\title{
Multiple Attribute Decision-Making Method Based upon Intuitionistic Fuzzy Partitioned Dual Maclaurin Symmetric Mean Operators
}

\author{
HongJuan Wang ${ }^{1} \cdot \mathrm{Yi} \mathrm{Liu}^{2} \cdot$ Fang $\mathrm{Liu}^{1,2} \cdot \mathrm{Jun} \mathrm{Lin}^{3} \mathbb{C}$
}

Received: 3 March 2021 / Accepted: 12 July 2021

(c) The Author(s) 2021

\begin{abstract}
We propound the idea of the partitioned dual Maclaurin symmetric mean (PDMSM) operator stimulated by the partitioned Maclaurin symmetric mean, suppose that we can partition overall attributes into some portions and the attributes are interrelated in the same portion, but the attributes are not interrelated in different portions. We can deal with decision-making issues using PDMSM operator in the intuitionistic fuzzy environment. We also analysis features and peculiar instance of the PDMSM operator. And, we extend the PDMSM operator to introduce the intuitionistic fuzzy partitioned dual Maclaurin symmetric mean operator and the weighted intuitionistic fuzzy partitioned dual Maclaurin symmetric mean operator. Then, we analysis several characteristics and peculiar instances of the developed operators. A new multiple attribute decisionmaking (MADM) approach grounded on the established weighted intuitionistic fuzzy partitioned dual Maclaurin symmetric mean operator is propounded; the MADM method is to choose the optimal alternative from several alternatives. Finally, we demonstrate the designed method is more general and effective than existing methods through comparative analysis.
\end{abstract}

Keywords Fuzzy sets $\cdot$ Intuitionistic fuzzy numbers $\cdot$ Partitioned dual Maclaurin symmetric mean $\cdot$ Multiple attribute decision making

\section{Introduction}

Multiple attribute decision-making (MADM) method is to choose the best alternative from viable alternatives; it has attracted more and more attention of people and been used in many aspects, such as financial risk management, location selection and so forth. Owing to the intricacy of

\section{Jun Lin}

junlin@hcnu.edu.cn

HongJuan Wang

whonjj@126.com

Yi Liu

liuyiyl@126.com

Fang Liu

lytb@163.com

1 School of Mathematics and Information Sciences, Neijiang Normal University, Neijiang 641000, Sichuan, China

2 Data Recovery Key Laboratory of Sichuan Province, Neijiang Normal University, Neijiang 641000, Sichuan, China

3 School of Big Data and Computer Science, Hechi University, Yizhou 546300, Guangxi, China
MADM issues and multiple attribute group decision-making (MAGDM) issues, using precise values to depict attribute values of alternatives is difficult. On this case, Zadeh [1] developed the fuzzy sets (FSs), which utilize the membership degree to estimate alternatives and have been studied extensively. However, the FSs have only the membership degree and are usual hard to depict much more complex fuzzy information. For purpose of surmounting above shorting, Atanassov [2] presented the intuitionistic fuzzy set (IFS); it is an extensiveness of FSs increasing non-membership degree and hesitant degree. Further, Xu and Yager [3] established the intuitionistic fuzzy numbers (IFNs) and operational rules of IFNs. In recent years, the IFNs have been diffusely used to cope with MADM issues, Garg et al. [4] introduced improved possibility degree method to rank intuitionistic fuzzy numbers, Joshi [5] established a new bi-parametric exponential information measure based on IFS, IFS combined with linguistic sets [6-8] and MAGDM issues [9-12]. Molodtsov [13] introduced soft sets, LEE [14] introduced bipolar valued fuzzy sets, and Mahmood [15] established bipolar valued soft sets; they are also generalizations of fuzzy sets and used in decision making [16, 17]. In order to resolve decision-making problems (DMPs), the 
aggregation operators (AOs) are one of the most popular tools, which combine all the input individual arguments into a single argument, many studies have been made [18-21]. The weighted arithmetic operators were introduced by $\mathrm{Xu}$ $[22]$ and $\mathrm{Xu}$ et al. $[3,23]$ proposed the weighted geometric operators based on the IFNs. In these AOs, the attributes are independent of each other. However, the relation of attribute cannot be ignored in DMPs. So, some operators consider that there is correlation between the aggregated arguments. In [24], Bonferroni developed the Bonferroni mean (BM); it presumes that correlation exists between any two arguments. Xu et al. [23] propounded the intuitionistic fuzzy BM (IFBM) operator and studied some desirable properties of IFBM operator. Xia et al. [25] put forward the geometric Bonferroni mean (GBM) by considering both the Bonferroni mean and the geometric mean for IFNs. Dutta et al. [26] introduced aggregation operators to fuse Extended Comparative LinguistiC Expressions with Symbolic Translation (ELICIT) information, namely ELICITBM, ELICITEBM and ELICITPBM. The Heronian mean (HM) which can capture correlation between two arguments was proposed by Sykora [27]. Liu et al. [28] introduced partitioned HM operator in linguistic intuitionistic fuzzy environment. Mo et al. [29] introduced Archimedean geometric Heronian mean operators in dual hesitant fuzzy environment.

The Maclaurin symmetric mean (MSM) was originally proposed by Maclaurin [30], which is a popular operator with aggregation function, it can capture the relation among multiple input arguments and then was extended by Detemple and Robertson [31], while BM and HM capture the relationship between two input arguments. The MSM operator was used to solve DMPs [32-35] with linguistic fuzzy set, used in IFS environment [36], used in q-rung interval-valued orthopair fuzzy environment [37], and used in dual hesitant fuzzy soft set environment [38]. Further, Qin and Liu [19] established the intuitionistic fuzzy MSM (IFMSM) and the weighted IFMSM (WIFMSM). In [39], Qin and Liu introduced the dual Maclaurin symmetric mean (DMSM) operator, which considers the interrelationship among multiple input arguments; there are some generalizations of DMSM. Shi and Xiao [40] established the reducible weighted dual Maclaurin symmetric mean (RWDMSM) operator, Qin and Liu [39] purposed some operators (the uncertain linguistic Choquet dual Maclaurin symmetric mean (ULCDMSM), the uncertain linguistic dual Maclaurin symmetric mean (ULDMSM), the uncertain linguistic weighted dual Maclaurin symmetric mean (ULWDMSM)), Darko et al. [41] established operators (dual hesitant fuzzy dual MSM and weighted dual hesitant fuzzy dual MSM operators), and Wang et al. [42] extended DMSM to interval-valued 2-tuple linguistic Pythagorean fuzzy numbers (IV2TLPFNs); these operators are able to reflect relationship among multiple attributes. However, in real decision-making situations, relation may not exist between attributes. For example, selecting a laptop from several laptops, we can consider following four attributes: basic requirements $\left(Z_{1}\right)$, customer comments $\left(Z_{2}\right)$, price comparison $\left(Z_{3}\right)$, appearance characteristic $\left(Z_{4}\right)$. These four attributes can be divided into two partitions: $P_{1}=\left\{Z_{1}, Z_{4}\right\}$ and $P_{2}=\left\{Z_{2}, Z_{3}\right\}$. We can know that the attribute $Z_{1}$ is interrelated to $Z_{4}$, they are all in $P_{1}$; the attribute $Z_{2}$ is interrelated to $Z_{3}$, they are all in $P_{2}$. But, there does not exist relationship between the partitions $P_{1}$ and $P_{2}$. Based on the condition, the partitioned Bonferroni mean (PBM) operator was presented by Dutta and Guha [43], Liu et al. [44] propounded the partitioned Maclaurin symmetric mean (PMSM) operator based on IFNs, these operators which suppose that total attributes are split into a few parts, and there exists relation between attributes in the same part and does not exist relation between attributes of diverse parts.

The DMSM operator considers that attributes are existing multiple relation. As previous study, it is easily found that generalizations of DMSM just only consider the case that attributes are relevant. But, in real DMPs, there may be not relationship between some attributes. Therefore, it is necessary that introduced the partitioned DMSM (PDMSM) to consider there is no relationship between some attributes. In this paper, our goals are: (1) to establish the PDMSM operator to surmount the drawback of the above discussion; (2) to put forward the intuitionistic fuzzy partitioned dual Maclaurin symmetric mean (IFPDMSM) operator and the weighted intuitionistic fuzzy partitioned dual Maclaurin symmetric mean (WIFPDMSM) operator; (3) to consider some properties, theorems and peculiar instances of the purposed operators; (4) to design a MADM methodology about the presented WIFPDMSM operator; and (5) to explain advantages of the established MADM approach.

The rest of the paper is described below. The Sect. 2 briefly gives notions of IFNs and DMSM. Besides, some properties of the DMSM are listed. In Sect. 3, we establish the PDMSM operator; we also give the features and particular case of the PDMSM operator. The Sect. 4 puts forward the IFPDMSM operator and the WIFPDMSM operator. The properties and peculiar instances of our introduced operators are too shown. The Sect. 5 designs a MADM methodology grounded on the put forward WIFPDMSM operator. Besides, giving an example and comparing the presented approach with other approaches to explain the presented MADM approach is more advantaged. The Sect. 6 is Conclusion and Future Studies. 


\section{Preliminaries}

Firstly, we tersely reviewed some basal conceptions such as definitions, operational laws and ranking method of IFNs. Then, the Maclaurin symmetric mean and the dual Maclaurin symmetric mean are brushed up on in this section.

\subsection{IFSs}

Definition 1 [2] Suppose $\tilde{Y}$ be a domain of discourse and $y \in \tilde{Y}$. Then an intuitionistic fuzzy set $\Gamma$ on $\tilde{Y}$ is indicated:

$\Gamma=\left\{\left\langle y, \rho_{\Gamma}(y), \tau_{\Gamma}(y)\right\rangle \mid y \in \tilde{Y}\right\}$

where $\rho_{\Gamma}$ and $\tau_{\Gamma}$ represent the membership degree (MD) and non-membership degree (NMD) to $\Gamma$, respectively, and $0 \leqslant \rho_{\Gamma}(y), \tau_{\Gamma}(y) \leqslant 1,0 \leqslant \rho_{\Gamma}(y)+\tau_{\Gamma}(y) \leqslant 1$.

The pair $\left(\rho_{\Gamma}(y), \tau_{\Gamma}(y)\right)$ is termed as intuitionistic fuzzy number (IFN) [22]. An IFN can be signified as $\theta=\left(\rho_{\theta}, \tau_{\theta}\right)$ for convenience, meeting $0 \leqslant \rho_{\theta}, \tau_{\theta} \leqslant 1$ and $0 \leqslant \rho_{\theta}+\tau_{\theta} \leqslant 1$.

Definition 2 [22] Let $\theta_{1}=\left(\rho_{1}, \tau_{1}\right)$ and $\theta_{2}=\left(\rho_{2}, \tau_{2}\right)$ be two IFNs. The operational laws of IFNs are represented as follows:

(1) $\theta_{1} \oplus \theta_{2}=\left(1-\left(1-\rho_{1}\right)\left(1-\rho_{2}\right), \tau_{1} \tau_{2}\right)$,

(2) $\theta_{1} \otimes \theta_{2}=\left(\rho_{1} \rho_{2}, 1-\left(1-\tau_{1}\right)\left(1-\tau_{2}\right)\right)$,

(3) $\lambda \theta_{1}=\left(1-\left(1-\rho_{1}\right)^{\lambda}, \tau_{1}^{\lambda}\right)$, where $\lambda$ is a real number and $\lambda>0$

(4) $\theta_{1}^{\lambda}=\left(\rho_{1}^{\lambda}, 1-\left(1-\tau_{1}\right)^{\lambda}\right)$, where $\lambda$ is a real number and $\lambda>0$.

By Definition 2, some operational laws of IFNs can be obtained easily and listed as follows:

(1) $\theta_{1} \oplus \theta_{2}=\theta_{2} \oplus \theta_{1}$,

(2) $\theta_{1} \otimes \theta_{2}=\theta_{2} \otimes \theta_{1}$,

(3) $\lambda\left(\theta_{1} \oplus \theta_{2}\right)=\lambda \theta_{1} \oplus \lambda \theta_{2}$, where $\lambda$ is a real number and $\lambda>0$

(4) $\left(\theta_{1} \otimes \theta_{2}\right)^{\lambda}=\theta_{1}^{\lambda} \otimes \theta_{2}^{\lambda}$, where $\lambda$ is a real number and $\lambda>0$

(5) $\lambda_{1} \theta_{1} \oplus \lambda_{2} \theta_{1}=\left(\lambda_{1}+\lambda_{2}\right) \theta_{1}$, where $\lambda_{1}, \lambda_{2}$ are real numbers and $\lambda_{1}, \lambda_{2}>0$,

(6) $\theta_{1}^{\lambda_{1}} \otimes \theta_{1}^{\lambda_{2}}=\theta_{1}^{\lambda_{1}+\lambda_{2}}$, where $\lambda_{1}, \lambda_{2}$ are real numbers and $\lambda_{1}, \lambda_{2}>0$.

In order to rank two IFNs, the score function and accuracy function are introduced.

Definition 3 [45] If $\theta=\left(\rho_{\theta}, \tau_{\theta}\right)$ be an IFN, then score function $S$ of the IFN $\theta$ is described:

$S(\theta)=\rho_{\theta}-\tau_{\theta}$ where $S(\theta) \in[0,1]$.

Definition 4 [46] If $\theta=\left(\rho_{\theta}, \tau_{\theta}\right)$ be an IFN, then accuracy function $H$ of the IFN $\theta$ is shown:

$H(\theta)=\rho_{\theta}+\tau_{\theta}$

where $H(\theta) \in[0,1]$.

Definition 5 [3] Suppose $\theta_{1}=\left(\rho_{1}, \tau_{1}\right)$ and $\theta_{2}=\left(\rho_{2}, \tau_{2}\right)$ be two IFNs, then

(1) if $S\left(\theta_{1}\right)>S\left(\theta_{2}\right)$, then $\theta_{1}>\theta_{2}$,

(2) if $S\left(\theta_{1}\right)=S\left(\theta_{2}\right)$, then if $H\left(\theta_{1}\right)>H\left(\theta_{2}\right)$, then $\theta_{1}>\theta_{2}$, if $H\left(\theta_{1}\right)=H\left(\theta_{2}\right)$, then $\theta_{1}=\theta_{2}$.

\subsection{The MSM Operator and DMSM Operator}

Definition 6 [30] For given nonnegative real numbers $z_{l}(l=1,2, \ldots, f)$, and $b=1,2, \ldots, f$. The MSM operator is given by:

$$
\begin{aligned}
& \operatorname{MSM}^{(b)}\left(z_{1}, z_{2}, \ldots, z_{f}\right) \\
& =\left(\frac{\sum_{1 \leqslant l_{1}<\cdots<l_{b} \leqslant f}\left(\prod_{q=1}^{b} z_{l_{q}}\right)}{C_{f}^{b}}\right)^{\frac{1}{b}}
\end{aligned}
$$

where $\left(l_{1}, l_{2}, \ldots, l_{b}\right)$ contains all the b-tuple combination of $(1,2, \ldots, f)$ and $C_{f}^{b}=\frac{f !}{b !(f-b) !}$.

Definition 7 [39] For given nonnegative real numbers $z_{l}(l=1,2, \ldots, f)$, and $b=1,2, \ldots, f$. The DMSM operator is described by:

$$
\begin{aligned}
& \operatorname{DMSM}^{(b)}\left(z_{1}, z_{2}, \ldots, z_{f}\right) \\
& =\frac{1}{b}\left(\prod_{1 \leqslant l_{1}<\cdots<l_{b} \leqslant f}\left(\sum_{q=1}^{b} z_{l_{q}}\right)^{\frac{1}{c_{f}^{b}}}\right)
\end{aligned}
$$

where $\left(l_{1}, l_{2}, \ldots, l_{b}\right)$ contains all the b-tuple combination of $(1,2, \ldots, f)$ and $C_{f}^{b}=\frac{f !}{b !(f-b) !}$.

The DMSM operator has the following properties [39]:

(1) if $z_{l}=z(l=1,2, \ldots, f)$, then $\operatorname{DMSM}^{(b)}\left(z_{1}, z_{2}, \ldots, z_{f}\right)=z$,

(2) if $z_{l} \leqslant a_{l}(l=1,2, \ldots, f)$, then

$$
\operatorname{DMSM}^{(b)}\left(z_{1}, z_{2}, \ldots, z_{f}\right) \leqslant \operatorname{DMSM}^{(b)}\left(a_{1}, a_{2}, \ldots, a_{f}\right),
$$

(3) $\min _{l}\left\{z_{l}\right\} \leqslant \operatorname{DMSM}^{(b)}\left(z_{1}, z_{2}, \ldots, z_{f}\right) \leqslant \max _{l}\left\{z_{l}\right\}$. 


\section{The PDMSM Operator}

The DMSM operator in the internal structure presents that input arguments are correlated to other input arguments. But in many real problems, input arguments may be partitioned into some parts and input argument be interrelated to other input arguments in the same part, interrelation does not exist between input arguments in different parts.

Let $Z_{l}(l=1,2, \ldots, f)$ be input arguments, and $z_{l}(l=1,2, \ldots, f)$ be values, where $z_{l}$ is the evaluating value of argument $Z_{l}, z_{l} \geqslant 0,1 \leqslant l \leqslant f$. Suppose that the collection $Z$ is divided into $c$ different partitions $P_{1}, P_{2}, \ldots, P_{c}$, meeting $P_{l} \cap P_{q}=\varnothing, l, q \in\{1,2, \ldots, c\}, l \neq q$ and $\bigcup_{t=1}^{c} P_{t}=Z$. We assume that the arguments are relevant in the identical partition and the arguments are irrelevant in diverse partitions.

In the following, we introduce the partitioned dual Maclaurin symmetric mean operator.

Definition 8 For given nonnegative real numbers $z_{l}(l=1,2, \ldots, f), c$ indicates the number of partitions, $b=1,2, \ldots, o_{t}, o_{t}$ is the amount of attributes in $P_{t}$. The PDMSM operator is defined:

$$
\begin{aligned}
& \operatorname{PDMSM}^{(b)}\left(z_{1}, z_{2}, \ldots, z_{f}\right) \\
& =\frac{1}{c} \sum_{t=1}^{c}\left(\frac{\prod_{1 \leqslant l_{1}<\cdots<l_{b} \leqslant o_{t}}\left(\sum_{q=1}^{b} z_{l_{q}}\right)^{\frac{1}{c_{o_{t}}^{b}}}}{b}\right)
\end{aligned}
$$

where $\left(l_{1}, l_{2}, \ldots, l_{b}\right)$ contains all the b-tuple combination of $\left(1,2, \ldots, o_{t}\right)$ and $C_{o_{t}}^{b}=\frac{o_{t} !}{b !\left(o_{t}-b\right) !}$.

Next, we will verify the characters of the introduced PDMSM operator.

Proposition 1 (Idempotency) Suppose $z_{1}=z_{2}=\cdots=z_{f}=z$, then

$\operatorname{PDMSM}^{(b)}\left(z_{1}, z_{2}, \ldots, z_{f}\right)=z$.

Proof By Definition 8 and $z_{1}=z_{2}=\cdots=z_{f}=z$, we know

$$
\begin{aligned}
& \operatorname{PDMSM}^{(b)}\left(z_{1}, z_{2}, \ldots, z_{f}\right) \\
& =\frac{1}{c} \sum_{t=1}^{c}\left(\frac{\prod_{\substack{1 \leqslant l_{1}<\ldots \\
<l_{b} \leqslant o_{t}}}\left(\sum_{q=1}^{b} z_{l_{q}}\right)^{\frac{1}{c_{o_{t}}}}}{b}\right) \\
& =\frac{1}{c} \sum_{t=1}^{c}\left(\frac{\prod_{\substack{1 \leqslant l_{1}<\ldots \\
<l_{b} \leqslant o_{t}}}\left(\sum_{q=1}^{b} z\right)^{\frac{1}{c_{o_{t}}}}}{b}\right)=\frac{1}{c} \sum_{t=1}^{c}\left(\frac{\sum_{q=1}^{b} z}{b}\right)=z,
\end{aligned}
$$

which completes the proof.

Proposition 2 (Monotonicity) Let $y_{l}, z_{l}(l=1,2, \ldots, f)$ be two groups of nonnegative real numbers and $y_{l} \leqslant z_{l}$, $l=1,2, \ldots, f$, then

$\operatorname{PDMSM}^{(b)}\left(y_{1}, y_{2}, \ldots, y_{f}\right) \leqslant \operatorname{PDMSM}^{(b)}\left(z_{1}, z_{2}, \ldots, z_{f}\right)$

when the parameter $b$ is fixed.

Proof By the definition of the PDMSM operator and $y_{l} \leqslant z_{l}$ for all $l$, we get $\sum_{q=1}^{b} y_{l_{q}} \leqslant \sum_{q=1}^{b} z_{l_{q}}$ and

$$
\begin{aligned}
& \left.\frac{1}{b} \prod_{1 \leqslant l_{1}<\cdots<l_{b} \leqslant o_{t}}\left(\sum_{q=1}^{b} y_{l_{q}}\right)^{\frac{1}{c_{o_{t}}^{b}}}\right) \\
& \quad \leqslant \frac{1}{b}\left(\prod_{1 \leqslant l_{1}<\cdots<l_{b} \leqslant o_{t}}\left(\sum_{q=1}^{b} z_{l_{q}}\right)^{\frac{1}{c_{o_{t}}^{b}}}\right) .
\end{aligned}
$$

Further, we obtain

$$
\begin{aligned}
& \frac{1}{c} \sum_{t=1}^{c}\left(\frac{\prod_{1 \leqslant l_{1}<\cdots<l_{b} \leqslant o_{t}}\left(\sum_{q=1}^{b} y_{l_{q}}\right)^{\frac{1}{c_{o_{t}}^{b}}}}{b}\right) \\
& \leqslant \frac{1}{c} \sum_{t=1}^{c}\left(\frac{\prod_{1 \leqslant l_{1}<\cdots<l_{b} \leqslant o_{t}}\left(\sum_{q=1}^{b} z_{l_{q}}\right)^{\frac{1}{c_{o_{t}}^{b}}}}{b}\right) .
\end{aligned}
$$

which completes the proof. 
Proposition 3 (Boundedness) Let $z_{l}(l=1,2, \ldots, f)$ be a collection of nonnegative real numbers, $z^{-}=\min _{l}\left\{z_{l}\right\}$ and $z^{+}=\max _{l}\left\{z_{l}\right\}$, then

$z^{-} \leqslant \operatorname{PDMSM}^{(b)}\left(z_{1}, z_{2}, \ldots, z_{f}\right) \leqslant z^{+}$.

Proof Because $z^{-}=\min _{l}\left\{z_{l}\right\} \leqslant z_{l}$, and owing to the Propositions 1 and 2, we can have

$$
\begin{aligned}
z^{-} & =\operatorname{PDMSM}^{(b)}\left(z^{-}, z^{-}, \ldots, z^{-}\right) \\
& \leqslant \operatorname{PDMSM}^{(b)}\left(z_{1}, z_{2}, \ldots, z_{f}\right) .
\end{aligned}
$$

In the same way, we can have

$$
\begin{aligned}
& \operatorname{PDMSM}^{(b)}\left(z_{1}, z_{2}, \ldots, z_{f}\right) \\
& \quad \leqslant \operatorname{PDMSM}^{(b)}\left(z^{+}, z^{+}, \ldots, z^{+}\right)=z^{+} .
\end{aligned}
$$

Therefore, $z^{-} \leqslant \operatorname{PDMSM}^{(b)}\left(z_{1}, z_{2}, \ldots, z_{f}\right) \leqslant z^{+}$.

Moreover, the effect of $b$ towards the established PDMSM operator can be studied.

Theorem 1 For given nonnegative real numbers $z_{l}(l=1,2, \ldots, f)$, then PDMSM operator monotonically increases with respect to the parameter $b$.

Proof By [39], we can have the DMSM operator inequality

$$
\operatorname{DMSM}^{(1)}\left(z_{1}, z_{2}, \ldots, z_{f}\right) \leqslant \operatorname{DMSM}^{(2)}\left(z_{1}, z_{2}, \ldots, z_{f}\right)
$$$$
\leqslant \cdots \leqslant \operatorname{DMSM}^{(f)}\left(z_{1}, z_{2}, \ldots, z_{f}\right) \text {. }
$$

Therefore, based on Eq. (6), we can obtain

$$
\begin{aligned}
& \operatorname{PDMSM}^{(1)}\left(z_{1}, z_{2}, \ldots, z_{f}\right) \\
& \leqslant \operatorname{PDMSM}^{(2)}\left(z_{1}, z_{2}, \ldots, z_{f}\right) \leqslant \cdots \\
& \leqslant \operatorname{PDMSM}_{t}^{\left(\min \left\{o_{t}\right\}\right)}\left(z_{1}, z_{2}, \ldots, z_{f}\right)
\end{aligned}
$$

where $b=1,2, \ldots, \min _{t}\left\{o_{t}\right\}$.

Theorem 2 For given nonnegative real numbers $z_{l}(l=1,2, \ldots, f)$, then

$$
\begin{aligned}
\min & \left\{\operatorname{PDMSM}^{(b)}\left(z_{1}, z_{2}, \ldots, z_{f}\right)\right\} \\
& =\operatorname{PDMSM}^{(1)}\left(z_{1}, z_{2}, \ldots, z_{f}\right)=\frac{1}{c} \sum_{t=1}^{c}\left(\prod_{l_{q}=1}^{o_{t}} z_{l_{q}}^{\frac{1}{o_{t}}}\right)
\end{aligned}
$$

and

$$
\begin{aligned}
\max & \left\{\operatorname{PDMSM}^{(b)}\left(z_{1}, z_{2}, \ldots, z_{f}\right)\right\} \\
& =\operatorname{PDMSM}_{t}^{\left(\min _{t}\left\{o_{t}\right\}\right)}\left(z_{1}, z_{2}, \ldots, z_{f}\right) .
\end{aligned}
$$

when $c=1$, the established PDMSM operator reduces to the DMSM operator [39], listed as:

$$
\begin{aligned}
\operatorname{PDMSM}^{(b)}\left(z_{1}, z_{2}, \ldots, z_{f}\right) \\
=\sum_{t=1}\left(\frac{\prod_{1 \leqslant l_{1}<\cdots<l_{b} \leqslant o_{1}}\left(\sum_{q=1}^{b} z_{l_{q}}\right)^{\frac{1}{c_{o_{1}}}}}{b}\right) \\
=\frac{1}{b}\left(\prod_{1 \leqslant l_{1}<\cdots<l_{b} \leqslant f}\left(\sum_{q=1}^{b} z_{l_{q}}\right)^{\frac{1}{c_{f}^{b}}}\right) .
\end{aligned}
$$

\section{The PDMSM Operator Based on the IFNs}

In the following, we will extend the PDMSM operator to propound the partitioned dual Maclaurin symmetric mean operator for IFNs and the weighted partitioned dual Maclaurin symmetric mean operator for IFNs. The detailed description is shown as follows.

\subsection{The IFPDMSM Operator}

Definition 9 For given IFNs $\theta_{l}=\left(\rho_{l}, \tau_{l}\right)(l=1,2, \ldots, f)$ partitioned into c distinct partitions $P_{1}, P_{2}, \ldots, P_{c}$, $b=1,2, \ldots, o_{t}, o_{t}$ indicates the amount of attributes in $P_{t}$. The IFPDMSM operator is described:

$$
\begin{aligned}
& \operatorname{IFPDMSM}^{(b)}\left(\theta_{1}, \theta_{2}, \ldots, \theta_{f}\right) \\
& \quad=\frac{1}{c}\left(\bigoplus_{t=1}^{c}\left(\frac{\underset{1 \leqslant l_{1}<\cdots<l_{b} \leqslant o_{t}}{\otimes}\left(\bigoplus_{q=1}^{b} \theta_{l_{q}}\right)^{\frac{1}{c_{o_{t}}}}}{b}\right)\right)
\end{aligned}
$$

where $\left(l_{1}, l_{2}, \ldots, l_{b}\right)$ contains overall the b-tuple combination of $\left(1,2, \ldots, o_{t}\right)$ and $C_{o_{t}}^{b}=\frac{o_{t} !}{b !\left(o_{t}-b\right) !}$.

Theorem 3 For given IFNs $\theta_{l}=\left(\rho_{l}, \tau_{l}\right)(l=1,2, \ldots, f)$. The aggregated result of formula (7) is still an IFN, described as follows: 
$\operatorname{IFPDMSM}^{(b)}\left(\theta_{1}, \theta_{2}, \ldots, \theta_{f}\right)$

$$
=\left(1-\left(\prod_{t=1}^{c}\left(1-\prod_{1 \leqslant l_{1}<\cdots<l_{b} \leqslant o_{t}}\left(1-\prod_{q=1}^{b}\left(1-\rho_{l_{q}}\right)\right)^{\frac{1}{c_{o_{t}}}}\right)^{\frac{1}{b}}\right)^{\frac{1}{c}},\left(\prod_{t=1}^{c}\left(1-\prod_{1 \leqslant l_{1}<\cdots<l_{b} \leqslant o_{t}}\left(1-\prod_{q=1}^{b} \tau_{l_{q}}\right)^{\frac{1}{c_{o_{t}}^{b}}}\right)^{\frac{1}{b}}\right) .\right.
$$

Proof By the operational laws of the IFNs, we can get

$$
\bigoplus_{q=1}^{b} \theta_{l_{q}}=\left(1-\prod_{q=1}^{b}\left(1-\rho_{l_{q}}\right), \prod_{q=1}^{b} \tau_{l_{q}}\right)
$$

and

$$
\begin{aligned}
& \left(\underset{q=1}{\stackrel{b}{\oplus}} \theta_{l_{q}}\right)^{\frac{1}{c_{o_{t}}^{b}}} \\
& \quad=\left(\left(1-\prod_{q=1}^{b}\left(1-\rho_{l_{q}}\right)\right)^{\frac{1}{c_{b_{t}}}}, 1-\left(1-\prod_{q=1}^{b} \tau_{l_{q}}\right)^{\frac{1}{c_{b_{t}}^{b}}}\right) .
\end{aligned}
$$

Then, we obtain

$$
\begin{aligned}
& \underset{1 \leqslant l_{1}<\cdots<l_{b} \leqslant o_{t}}{\otimes}\left(\bigoplus_{q=1}^{b} \theta_{l_{q}}\right)^{\frac{1}{c_{o_{t}}}} \\
& =\prod_{\substack{1 \leqslant l_{1}<\ldots \\
<l_{b} \leqslant o_{t}}}\left(1-\prod_{q=1}^{b}\left(1-\rho_{l_{q}}\right)\right)^{\frac{1}{c_{o_{t}}^{b}}}, \\
& \\
& \left.\quad \prod_{\substack{1 \leqslant l_{1}<\cdots \\
<l_{b} \leqslant o_{t}}}\left(1-\prod_{q=1}^{b} \tau_{l_{q}}\right)^{\frac{1}{c_{o_{t}}^{b}}}\right)
\end{aligned}
$$

and

$$
\begin{aligned}
& \frac{1}{b}\left(\underset{1 \leqslant l_{1}<\cdots<l_{b} \leqslant o_{t}}{\otimes}\left(\bigoplus_{q=1}^{b} \theta_{l_{q}}\right)^{\frac{1}{c_{o_{t}}^{b}}}\right) \\
& =\left(1-\left(1-\prod_{\substack{1 \leqslant l_{1}<\ldots . \\
<l_{b} \leqslant o_{t}}}\left(1-\prod_{q=1}^{b}\left(1-\rho_{l_{q}}\right)\right)^{\frac{1}{c_{o_{t}}^{b}}}\right)^{\frac{1}{b}}\right. \text {, } \\
& \left.\left(1-\prod_{1 \leqslant l_{1}<\cdots<l_{b} \leqslant o_{t}}\left(1-\prod_{q=1}^{b} \tau_{l_{q}}\right)^{\frac{1}{C_{o_{t}}}}\right)^{\frac{1}{b}}\right) \text {. }
\end{aligned}
$$

Therefore, we can get 
$\operatorname{IFPDMSM}^{(b)}\left(\theta_{1}, \theta_{2}, \ldots, \theta_{f}\right)=\left(1-\left(\prod_{t=1}^{c}\left(1-\prod_{1 \leqslant l_{1}<\cdots<l_{b} \leqslant o_{t}}\left(1-\prod_{q=1}^{b}\left(1-\rho_{l_{q}}\right)\right)^{\frac{1}{c_{o_{t}}}}\right)^{\frac{1}{b}}\right)^{\frac{1}{c}}\right.$,

$$
\left.\left(\prod_{t=1}^{c}\left(1-\prod_{1 \leqslant l_{1}<\cdots<l_{b} \leqslant o_{t}}\left(1-\prod_{q=1}^{b} \tau_{l_{q}}\right)^{\frac{1}{c_{o_{t}}}}\right)^{\frac{1}{b}}\right)^{\frac{1}{c}}\right) \text {. }
$$

Next,

$0 \leqslant 1-\left(\prod_{t=1}^{c}\left(1-\prod_{\substack{1 \leqslant l_{1}<\ldots \\<l_{b} \leqslant o_{t}}}\left(1-\prod_{q=1}^{b}\left(1-\rho_{l_{q}}\right)\right)^{\frac{1}{c_{o_{t}}}}\right)^{\frac{1}{b}}\right)^{\frac{1}{c}} \leqslant 1$ $0 \leqslant 1-\left(\prod_{t=1}^{c}\left(1-\prod_{\substack{1 \leqslant l_{1}<\ldots \\<l_{b} \leqslant o_{t}}}\left(1-\prod_{q=1}^{b}\left(1-\rho_{l_{q}}\right)\right)^{\frac{1}{c_{o_{t}}}}\right)^{\frac{1}{b}}\right)^{\frac{1}{c}}$ $+\left(\prod_{t=1}^{c}\left(1-\prod_{1 \leqslant l_{1}<\cdots<l_{b} \leqslant o_{t}}\left(1-\prod_{q=1}^{b} \tau_{l_{q}}\right)^{\frac{1}{c_{o_{t}}^{b}}}\right)^{\frac{1}{b}}\right)^{\frac{1}{c}}$

$0 \leqslant\left(\prod_{t=1}^{c}\left(1-\prod_{\substack{1 \leqslant l_{1}<\ldots \\<l_{b} \leqslant o_{t}}}\left(1-\prod_{q=1}^{b} \tau_{l_{q}}\right)^{\frac{1}{c_{o_{t}}}}\right)^{\frac{1}{b}}\right)^{\frac{1}{c}} \leqslant 1$. $\leqslant 1-\left(\prod_{t=1}^{c}\left(1-\prod_{\substack{1 \leqslant l_{1}<\ldots \\<l_{b} \leqslant o_{t}}}\left(1-\prod_{q=1}^{b} \tau_{l_{q}}\right)^{\frac{1}{c_{o_{t}}}}\right)^{\frac{1}{b}}\right)^{\frac{1}{c}}$

Because $0 \leqslant \rho_{l_{q}} \leqslant 1,0 \leqslant \tau_{l_{q}} \leqslant 1$ and $0 \leqslant \rho_{l_{q}}+\tau_{l_{q}} \leqslant 1$, we $+\left(\prod_{t=1}^{c}\left(1-\prod_{1 \leqslant l_{1}<\cdots<l_{b} \leqslant o_{t}}\left(1-\prod_{q=1}^{b} \tau_{l_{q}}\right)^{\frac{1}{c_{o_{t}}}}\right)^{\frac{1}{b}}\right)^{\frac{1}{c}}$ $=1$.

Thus, we complete the proof.

Also, we can get the IFPDMSM operator has following desirable properties. 
Proposition 4 (Idempotency) If $\theta_{l}(l=1,2, \ldots, f)$ are equal, i.e., $\theta_{l}=\left(\rho_{l}, \tau_{l}\right)=\theta=(\rho, \tau)(l=1,2, \ldots, f)$. Then,

$\operatorname{IFPDMSM}^{(b)}\left(\theta_{1}, \theta_{2}, \ldots, \theta_{f}\right)=\theta$.

Proof On the ground of Eq. (8), we can have

$$
\begin{aligned}
& \operatorname{IFPDMSM}^{(b)}\left(\theta_{1}, \theta_{2}, \ldots, \theta_{f}\right) \\
& =\left(1-\left(\prod_{t=1}^{c}\left(1-\prod_{\substack{1 \leqslant l_{1}<\ldots \\
<l_{b} \leqslant o_{t}}}\left(1-\prod_{q=1}^{b}\left(1-\rho_{l_{q}}\right)\right)^{\frac{1}{c_{o_{t}}}}\right)^{\frac{1}{b}}\right),\left(\prod_{t=1}^{c}\left(1-\prod_{\substack{\frac{1}{c} \\
1 \leqslant l_{1}<\ldots \\
<l_{b} \leqslant o_{t}}}\left(1-\prod_{q=1}^{b} \tau_{l_{q}}\right)^{\frac{1}{c_{o_{t}}}}\right)^{\frac{1}{b}}\right)^{\frac{1}{c}}\right) \\
& =\left(1-\left(\prod_{t=1}^{c}\left(1-\prod_{\substack{1 \leqslant l_{1}<\ldots \\
<l_{b} \leqslant o_{t}}}\left(1-\prod_{q=1}^{b}(1-\rho)\right)^{\frac{1}{c_{t}}}\right)^{\frac{1}{b}}\right)^{\frac{1}{c}},\left(\prod_{t=1}^{c}\left(1-\prod_{\substack{1 \leqslant l_{1}<\ldots \\
<l_{b} \leqslant o_{t}}}\left(1-\prod_{q=1}^{b} \tau\right)^{\frac{1}{c_{o_{t}}}}\right)^{\frac{1}{b}}\right)^{\frac{1}{c}}\right) \\
& =\left(1-\left(\prod_{t=1}^{c}\left(1-\left(\left(1-(1-\rho)^{b}\right)^{\frac{1}{C_{o_{t}}}}\right)^{C_{o_{t}}^{b}}\right)^{\frac{1}{b}}\right)^{\frac{1}{c}},\left(\prod_{t=1}^{c}\left(1-\left(\left(1-\tau^{b}\right)^{\frac{1}{C_{o_{t}}^{b}}}\right)^{C_{o_{t}}^{b}}\right)^{\frac{1}{b}}\right)^{\frac{1}{c}}\right) \\
& =\left(1-\left(\prod_{t=1}^{c}\left((1-\rho)^{b}\right)^{\frac{1}{b}}\right)^{\frac{1}{c}},\left(\prod_{t=1}^{c}\left(\tau^{b}\right)^{\frac{1}{b}}\right)^{\frac{1}{c}}\right)=(\rho, \tau)=\theta .
\end{aligned}
$$

Proposition 5 (Commutativity) For given IFNs $\theta_{l}=\left(\rho_{l}, \tau_{l}\right)$ and $\ddot{\theta}_{l}=\left(\ddot{\rho}_{l}, \ddot{\tau}_{l}\right)(l=1,2, \ldots, f)$. Suppose $\ddot{\theta}_{l}=\left(\ddot{\rho}_{l}, \ddot{\tau}_{l}\right)$ is any permutation of $\theta_{l}=\left(\rho_{l}, \tau_{l}\right)$ for all $l$, then 
$\operatorname{IFPDMSM}^{(b)}\left(\theta_{1}, \theta_{2}, \ldots, \theta_{f}\right)$

$$
=\operatorname{IFPDMSM}^{(b)}\left(\ddot{\theta}_{1}, \ddot{\theta}_{2}, \ldots, \ddot{\theta}_{f}\right) \text {. }
$$

Proof According to Eq. (8), we can get

$$
\begin{aligned}
& \operatorname{IFPDMSM}^{(b)}\left(\theta_{1}, \theta_{2}, \ldots, \theta_{f}\right) \\
& =\left(1-\left(\prod_{t=1}^{c}\left(1-\prod_{\substack{1 \leqslant l_{1}<\ldots \\
<l_{b} \leqslant o_{t}}}\left(1-\prod_{q=1}^{b}\left(1-\rho_{l_{q}}\right)\right)^{\frac{1}{c_{o_{t}}}}\right)^{\frac{1}{b}}\right)^{\frac{1}{c}},\right. \\
& \left.\left(\prod_{t=1}^{c}\left(1-\prod_{\substack{1 \leqslant l_{1}<\ldots \\
<l_{b} \leqslant o_{t}}}\left(1-\prod_{q=1}^{b} \tau_{l_{q}}\right)^{\frac{1}{c_{t_{t}}}}\right)^{\frac{1}{b}}\right)^{\frac{1}{c}}\right) .
\end{aligned}
$$

$\operatorname{IFPDMSM}^{(b)}\left(\ddot{\theta}_{1}, \ddot{\theta}_{2}, \ldots, \ddot{\theta}_{f}\right)$

$$
\begin{aligned}
& =\left(1-\left(\prod_{t=1}^{c}\left(1-\prod_{\substack{1 \leqslant l_{1}<\cdots \\
<l_{b} \leqslant o_{t}}}\left(1-\prod_{q=1}^{b}\left(1-\ddot{\rho}_{l_{q}}\right)\right)^{\frac{1}{c_{o_{t}}}}\right)^{\frac{1}{b}}\right)^{\frac{1}{c}}\right. \\
& \left(\prod_{t=1}^{c}\left(1-\prod_{\substack{1 \leqslant l_{1}<\ldots \\
<l_{b} \leqslant o_{t}}}\left(1-\prod_{q=1}^{b} \ddot{\tau}_{l_{q}}\right)^{\frac{1}{c_{o_{t}}}}\right)^{\frac{1}{b}}\right) .
\end{aligned}
$$

Because $\ddot{\theta}_{l}=\left(\ddot{\rho}_{l}, \ddot{\tau}_{l}\right)$ is any permutation of $\theta_{l}=\left(\rho_{l}, \tau_{l}\right)$ for all $l$, we have

$$
\begin{aligned}
& \operatorname{IFPDMSM}^{(b)}\left(\theta_{1}, \theta_{2}, \ldots, \theta_{f}\right) \\
& \quad=\operatorname{IFPDMSM}^{(b)}\left(\ddot{\theta}_{1}, \ddot{\theta}_{2}, \ldots, \ddot{\theta}_{f}\right) .
\end{aligned}
$$

Proposition 6 (Monotonicity) Let $\theta_{l}=\left(\rho_{l}, \tau_{l}\right)$ and $\ddot{\theta}_{l}=\left(\ddot{\rho}_{l}, \ddot{\tau}_{l}\right)(l=1,2, \ldots, f)$ be arbitrary two collections of IFNs, satisfying $\rho_{l} \geqslant \ddot{\rho}_{l}, \tau_{l} \leqslant \ddot{\tau}_{l}$ for all $l$, then
$\operatorname{IFPDMSM}^{(b)}\left(\theta_{1}, \theta_{2}, \ldots, \theta_{f}\right)$

$$
\geqslant \operatorname{IFPDMSM}^{(b)}\left(\ddot{\theta}_{1}, \ddot{\theta}_{2}, \ldots, \ddot{\theta}_{f}\right)
$$

when the parameter $b$ is fixed.

Proof Suppose that $\operatorname{IFPDMSM}^{(b)}\left(\theta_{1}, \theta_{2}, \ldots, \theta_{f}\right)=\theta=(\rho, \tau)$ and $\operatorname{IFPDMSM}^{(b)}\left(\ddot{\theta}_{1}, \ddot{\theta}_{2}, \ldots, \ddot{\theta}_{f}\right)=\ddot{\theta}=(\ddot{\rho}, \ddot{\tau})$, then

$\rho=1-\left(\prod_{t=1}^{c}\left(1-\prod_{\substack{1 \leqslant l_{1}<\ldots \\<l_{b} \leqslant o_{t}}}\left(1-\prod_{q=1}^{b}\left(1-\rho_{l_{q}}\right)\right)^{\frac{1}{c_{o_{t}}}}\right)^{\frac{1}{b}}\right)^{\frac{1}{c}}$,
$\ddot{\rho}=1-\left(\prod_{t=1}^{c}\left(1-\prod_{\substack{1 \leqslant l_{1}<\ldots \\<l_{b} \leqslant o_{t}}}\left(1-\prod_{q=1}^{b}\left(1-\ddot{\rho}_{l_{q}}\right)\right)^{\frac{1}{c_{o_{t}}}}\right)^{\frac{1}{b}}\right)^{\frac{1}{c}}$

$\tau=\left(\prod_{t=1}^{c}\left(1-\prod_{\substack{1 \leqslant l_{1}<\ldots \\<l_{b} \leqslant o_{t}}}\left(1-\prod_{q=1}^{b} \tau_{l_{q}}\right)^{\frac{1}{c_{o_{t}}}}\right)^{\frac{1}{b}}\right)^{\frac{1}{c}}$,

$\ddot{\tau}=\left(\prod_{t=1}^{c}\left(1-\prod_{\substack{1 \leqslant l_{1}<\ldots \\<l_{b} \leqslant o_{t}}}\left(1-\prod_{q=1}^{b} \ddot{\tau}_{l_{q}}\right)^{\frac{1}{c_{o_{t}}}}\right)^{\frac{1}{b}}\right)^{\frac{1}{c}}$.

Because $\rho_{l} \geqslant \ddot{\rho}_{l}, \tau_{l} \leqslant \ddot{\tau}_{l}$, then 


$$
\begin{aligned}
& 1-\left(\prod_{t=1}^{c}\left(1-\prod_{\substack{1 \leqslant l_{1}<\ldots \\
<l_{b} \leqslant o_{t}}}\left(1-\prod_{q=1}^{b}\left(1-\rho_{l_{q}}\right)\right)^{\frac{1}{c_{o_{t}}}}\right)^{\frac{1}{b}}\right)^{\frac{1}{c}} \\
& \geqslant 1-\left(\prod_{t=1}^{c}\left(1-\prod_{\substack{1 \leqslant l_{1}<\ldots \\
<l_{b} \leqslant o_{t}}}\left(1-\prod_{q=1}^{b}\left(1-\ddot{\rho}_{l_{q}}\right)\right)^{\frac{1}{c_{o_{t}}}}\right)^{\frac{1}{b}}\right)^{\frac{1}{c}}, \\
& \left(\prod_{t=1}^{c}\left(1-\prod_{\substack{1 \leqslant l_{1}<\ldots \\
<l_{b} \leqslant o_{t}}}\left(1-\prod_{q=1}^{b} \tau_{l_{q}}\right)^{\frac{1}{c_{o_{t}}}}\right)^{\frac{1}{b}}\right)^{\frac{1}{c}} \\
& \leqslant\left(\prod_{t=1}^{c}\left(1-\prod_{\substack{1 \leqslant l_{1}<\ldots \\
<l_{b} \leqslant o_{t}}}\left(1-\prod_{q=1}^{b} \ddot{\tau}_{l_{q}}\right)^{\frac{1}{c_{o_{t}}}}\right)^{\frac{1}{b}}\right)^{\frac{1}{c}} .
\end{aligned}
$$

That is $\rho \geqslant \ddot{\rho}$ and $\tau \leqslant \ddot{\tau}$, then $\theta \geqslant \ddot{\theta}$. Therefore,

$$
\begin{aligned}
& \operatorname{IFPDMSM}^{(b)}\left(\theta_{1}, \theta_{2}, \ldots, \theta_{f}\right) \\
& \quad \geqslant \operatorname{IFPDMSM}^{(b)}\left(\ddot{\theta}_{1}, \ddot{\theta}_{2}, \ldots, \ddot{\theta}_{f}\right) .
\end{aligned}
$$

Proposition 7 (Boundedness) For given IFNs $\theta_{l}=\left(\rho_{l}, \tau_{l}\right)(l=1,2, \ldots, f), \quad \theta^{-}=\min _{l}\left\{\theta_{l}\right\} \quad$ and $\theta^{+}=\max _{l}\left\{\theta_{l}\right\}, l=1,2, \ldots, f$, then

$\theta^{-} \leqslant \operatorname{IFPDMSM}^{(b)}\left(\theta_{1}, \theta_{2}, \ldots, \theta_{f}\right) \leqslant \theta^{+}$.

Proof Owing to $\theta^{-}=\min _{l}\left\{\theta_{l}\right\} \leqslant \theta_{l}$, and Propositions 4 and 6 , we have

$$
\begin{aligned}
\theta^{-} & =\operatorname{IFPDMSM}^{(b)}\left(\theta^{-}, \theta^{-}, \ldots, \theta^{-}\right) \\
& \leqslant \operatorname{IFPDMSM}^{(b)}\left(\theta_{1}, \theta_{2}, \ldots, \theta_{f}\right)
\end{aligned}
$$

and

$$
\begin{aligned}
& \operatorname{IFPDMSM}^{(b)}\left(\theta_{1}, \theta_{2}, \ldots, \theta_{f}\right) \\
& \quad \leqslant \operatorname{IFPDMSM}^{(b)}\left(\theta^{+}, \theta^{+}, \ldots, \theta^{+}\right)=\theta^{+} .
\end{aligned}
$$

Thus, we have $\theta^{-} \leqslant \operatorname{IFPDMSM}^{(b)}\left(\theta_{1}, \theta_{2}, \ldots, \theta_{f}\right) \leqslant \theta^{+}$.

Furthermore, we study the impact of $b$ for the IFPDMSM operator and an example.

Lemma 1 [47] Suppose $y_{l}>0, z_{l}>0(l=1,2, \ldots, f)$ meeting $\sum_{l=1}^{f} z_{l}=1$, then $\prod_{l=1}^{f} y_{l}^{z_{l}} \leqslant \sum_{l=1}^{f} y_{l} z_{l}$.

Theorem 4 Let $\theta_{l}=\left(\rho_{l}, \tau_{l}\right)(l=1,2, \ldots, f)$ be a group of IFNs, and $b=1,2, \ldots, \min _{t}\left\{o_{t}\right\}$. The IFPDMSM operator monotonically increases with respect to the parameter $b$.

Proof Based on Theorem 3, we have

$$
\begin{aligned}
& \operatorname{IFPDMSM}^{(b)}\left(\theta_{1}, \theta_{2}, \ldots, \theta_{f}\right) \\
& =\left(1-\left(\prod_{t=1}^{c}\left(1-\prod_{\substack{1 \leqslant l_{1}<\ldots \\
<l_{b} \leqslant o_{t}}}\left(1-\prod_{q=1}^{b}\left(1-\rho_{l_{q}}\right)\right)^{\frac{1}{c_{o_{t}}}}\right)^{\frac{1}{b}}\right)^{\frac{1}{c}},\right. \\
& \left.\left(\prod_{t=1}^{c}\left(1-\prod_{\substack{1 \leqslant l_{1}<\ldots \\
<l_{b} \leqslant o_{t}}}\left(1-\prod_{q=1}^{b} \tau_{l_{q}}\right)^{\frac{1}{c_{o_{t}}}}\right)^{\frac{1}{b}}\right)^{\frac{1}{c}}\right) .
\end{aligned}
$$

Assume that

$$
\begin{aligned}
& G(b)=1-\left(\prod_{t=1}^{c}\left(1-\prod_{\substack{1 \leqslant l_{1}<\ldots \\
<l_{b} \leqslant o_{t}}}\left(1-\prod_{q=1}^{b}\left(1-\rho_{l_{q}}\right)\right)^{\frac{1}{c_{o_{t}}^{b}}}\right)^{\frac{1}{b}}\right)^{\frac{1}{c}}, \\
& H(b)=\left(\prod_{t=1}^{c}\left(1-\prod_{\substack{1 \leqslant l_{1}<\ldots \\
<l_{b} \leqslant o_{t}}}\left(1-\prod_{q=1}^{b} \tau_{l_{q}}\right)^{\frac{1}{c_{o_{t}}^{b}}}\right)^{\frac{1}{b}}\right)^{\frac{1}{c}} .
\end{aligned}
$$

Next, we prove function $G(b)$ monotonically increases as $b$ increases. Based on the dual Maclaurin inequality and Lemma 1, we have 


$$
\begin{aligned}
& \prod_{1 \leqslant l_{1}<\cdots<l_{b} \leqslant o_{t}}\left(1-\prod_{q=1}^{b}\left(1-\rho_{l_{q}}\right)\right)^{\frac{1}{c_{o_{t}}}} \\
& \leqslant \sum_{1 \leqslant l_{1}<\cdots<l_{b} \leqslant o_{t}} \frac{1-\prod_{q=1}^{b}\left(1-\rho_{l_{q}}\right)}{C_{o_{t}}^{b}} \\
& \Rightarrow 1-\prod_{\substack{1 \leqslant l_{1}<\cdots \\
<l_{b} \leqslant o_{t}}}\left(1-\prod_{q=1}^{b}\left(1-\rho_{l_{q}}\right)\right)^{\frac{1}{c_{t_{t}}}} \\
& \geqslant
\end{aligned}
$$

Therefore,

$$
\begin{aligned}
& G(b)=1-\left(\prod_{t=1}^{c}\left(1-\prod_{\substack{1 \leqslant l_{1}<\ldots \\
<l_{b} \leqslant o_{t}}}\left(1-\prod_{q=1}^{b}\left(1-\rho_{l_{q}}\right)\right)^{\frac{1}{c_{o_{t}}}}\right)^{\frac{1}{b}}\right)^{\frac{1}{c}} \\
& \leqslant 1-\left(\prod_{t=1}^{c}\left(\sum_{1 \leqslant l_{1}<\cdots<l_{b} \leqslant o_{t}} \frac{\prod_{q=1}^{b}\left(1-\rho_{l_{q}}\right)}{C_{o_{t}}^{b}}\right)^{\frac{1}{b}}\right)^{\frac{1}{c}} .
\end{aligned}
$$

In the following, we take the proof by contradiction methodology. Assume $G(b)$ is monotonically decreasing as $b$ increases, then $G\left(\min _{t}\left\{o_{t}\right\}\right)<\cdots<G(2)<G(1)$ and we can have

$$
\begin{aligned}
G(1) \leqslant 1-\left(\prod_{t=1}^{c}\left(\sum_{\substack{1 \leqslant l_{1}<\cdots \\
<l_{b} \leqslant o_{t}}} \frac{\prod_{q=1}^{1}\left(1-\rho_{l_{q}}\right)}{C_{o_{t}}^{1}}\right)\right)^{\frac{1}{c}} \\
=1-\left(\prod_{t=1}^{c} \frac{\sum_{l_{q}=1}^{o_{t}}\left(1-\rho_{l_{q}}\right)}{o_{t}}\right)^{\frac{1}{c}} .
\end{aligned}
$$

In addition, we assume that $o_{t}=o$, then we can have 


$$
\begin{aligned}
& G\left(\min _{t}\left\{o_{t}\right\}\right)=G(o)=1-\left(\prod_{t=1}^{c}\left(1-\prod_{\substack{1 \leqslant l_{1}<\ldots \\
<l_{b} \leqslant o}}\left(1-\prod_{q=1}^{o}\left(1-\rho_{l_{q}}\right)\right)^{\frac{1}{C_{o}^{o}}}\right)^{\frac{1}{o}}\right)^{\frac{1}{c}} \\
& =1-\left(\prod_{t=1}^{c}\left(\prod_{q=1}^{o}\left(1-\rho_{l_{q}}\right)\right)^{\frac{1}{o}}\right)^{\frac{1}{c}} .
\end{aligned}
$$

According to the assumption $G(o)<G(1)$, we can get

$$
\begin{aligned}
G(o) & =1-\left(\prod_{t=1}^{c}\left(\prod_{l_{q}=1}^{o}\left(1-\rho_{l_{q}}\right)\right)^{\frac{1}{o}}\right)^{\frac{1}{c}}<G(1) \\
& \left.\left.\leqslant 1-\left(\prod_{t=1}^{c} \frac{\left.\sum_{l_{q}=1}^{o_{t}}\left(1-\rho_{l_{q}}\right)\right)^{\frac{1}{c}}}{o_{t}}\right)^{\frac{1}{c}}\right)^{\frac{1}{c}}\left(1-\rho_{l_{q}}\right)\right)^{c} \sum_{l_{q}=1}^{o}\left(1-\rho_{l_{q}}\right) \\
& =1-\left(\prod_{l_{q}=1}^{o} .\right. \\
& \Rightarrow\left(\prod_{l_{q}=1}^{o}\left(1-\rho_{l_{q}}\right)\right)^{\frac{1}{o}}>\frac{\sum_{l}}{o}
\end{aligned}
$$

Obviously, $\left(\prod_{l_{q}=1}^{o}\left(1-\rho_{l_{q}}\right)\right)^{\frac{1}{o}}>\frac{\sum_{l_{q}=1}^{o}\left(1-\rho_{l_{q}}\right)}{o}$ is contradiction to the Lemma 1 . Thus, as the $b$ increases, function $G(b)$ is monotonically increasing. In the same way, we can prove function $H(b)$ is monotonically decreasing as the $b$ increases.

In accordance with the previous analysis, we can have

$$
\begin{aligned}
& \operatorname{IFPDMSM}^{(b)}\left(\theta_{1}, \theta_{2}, \ldots, \theta_{f}\right) \\
& \quad<\operatorname{IFPDMSM}^{(b+1)}\left(\theta_{1}, \theta_{2}, \ldots, \theta_{f}\right) .
\end{aligned}
$$

Therefore, the IFPDMSM operator monotonically increases with respect to the parameter $b$.

Theorem 5 Let $\theta_{l}=\left(\rho_{l}, \tau_{l}\right)(l=1,2, \ldots, f)$ be a group of IFNs, and $b=1,2, \ldots, \min _{t}\left\{o_{t}\right\}$. Then

$$
\begin{aligned}
\min & \left\{\operatorname{IFPDMSM}^{(b)}\left(\theta_{1}, \theta_{2}, \ldots, \theta_{f}\right)\right\} \\
& =\operatorname{IFPDMSM}^{(1)}\left(\theta_{1}, \theta_{2}, \ldots, \theta_{f}\right) \\
& =\left(1-\left(\prod_{t=1}^{c}\left(1-\prod_{l_{q}=1}^{o_{t}}\left(\rho_{l_{q}}\right)^{\frac{1}{o_{t}}}\right)\right)^{\frac{1}{c}},\right. \\
& \left.\left(\prod_{t=1}^{c}\left(1-\prod_{l_{q}=1}^{o_{t}}\left(1-\tau_{l_{q}}\right)^{\frac{1}{o_{t}}}\right)\right)^{\frac{1}{c}}\right)
\end{aligned}
$$

and

$$
\begin{aligned}
\max & \left\{\operatorname{IFPDMSM}^{(b)}\left(\theta_{1}, \theta_{2}, \ldots, \theta_{f}\right)\right\} \\
& =\operatorname{IFPDMSM}_{t}^{\left(\min _{t}\left\{o_{t}\right\}\right)}\left(\theta_{1}, \theta_{2}, \ldots, \theta_{f}\right) .
\end{aligned}
$$

$\begin{array}{lllllllllllllll}E & x & a & m & p & l & e & 1 & \mathrm{~L} & \mathrm{e} & \mathrm{t}\end{array}$ $\theta_{1}=(0.5,0.3), \theta_{2}=(0.4,0.4), \theta_{3}=(0.6,0.2), \theta_{4}=(0.7,0.2)$ and $\theta_{5}=(0.3,0.4)$ be five IFNs. Suppose these five IFNs are partitioned into two groups $P_{1}=\left\{\theta_{1}, \theta_{3}, \theta_{5}\right\}$ and $P_{2}=\left\{\theta_{2}, \theta_{4}\right\}$. Here we use the IFPDMSM to aggregate these five IFNs. In general, we let $b=2$, then

$$
\begin{aligned}
&\left(\prod_{t=1}^{2}\left(1-\prod_{1 \leqslant l_{1}<l_{2} \leqslant o_{t}}\left(1-\prod_{q=1}^{2} \tau_{l_{q}}\right)^{\frac{1}{c_{o_{t}}}}\right)^{\frac{1}{2}}\right)^{\frac{1}{2}} \\
&=\left(\left(1-(1-0.3 \times 0.2)^{\frac{1}{3}} \times(1-0.3 \times 0.4)^{\frac{1}{3}}\right.\right. \\
&\left.\left.\quad \times(1-0.2 \times 0.4)^{\frac{1}{3}}\right)^{\frac{1}{2}} \times(1-(1-0.4 \times 0.2))^{\frac{1}{2}}\right)^{\frac{1}{2}} \\
&= 0.2888 .
\end{aligned}
$$

Similarly, 
$1-\left(\prod_{t=1}^{2}\left(1-\prod_{\substack{1 \leqslant l_{1} \\<l_{2} \leqslant o_{t}}}\left(1-\prod_{q=1}^{2}\left(1-\rho_{l_{q}}\right)\right)^{\frac{1}{c_{o_{t}}}}\right)^{\frac{1}{2}}\right)^{\frac{1}{2}}$

$=0.5265$.

$\begin{array}{llllllllllll}\mathrm{T} & \mathrm{h} & \mathrm{e} & \mathrm{r} & \mathrm{e} & \mathrm{f} & \mathrm{o} & \mathrm{r} & \mathrm{e} & \text {, }\end{array}$ $\operatorname{IFPDMSM}^{(2)}\left(\theta_{1}, \theta_{2}, \theta_{3}, \theta_{4}, \theta_{5}\right)=(0.5265,0.2888)$.

\subsection{The WIFPDMSM Operator}

In Definition 9, the IFPDMSM operator does not view the importance of each input element. But, input elements always have diverse significance in practical DMPs, so we should consider that the weight of elements may be different. Following, We introduce the WIFPDMSM operator for IFNs which considers the importance of elements.

Definition 10 For given IFNs $\theta_{l}=\left(\rho_{l}, \tau_{l}\right)(l=1,2, \ldots, f)$ partitioned into c distinct partitions $P_{1}, P_{2}, \ldots, P_{c}$ and the weight of IFN $\theta_{l}(l=1,2, \ldots, f)$ is $\omega_{l}$, meeting $\omega_{l} \in[0,1]$ and $\sum_{l=1}^{f} \omega_{l}=1, b=1,2, \ldots, o_{t}, o_{t}$ signifies the number of attributes in $P_{t}$. Then the WIFPDMSM operator of the $\theta_{l}(l=1,2, \ldots, f)$ is defined as:

$$
\begin{aligned}
& \operatorname{WIFPDMSM}^{(b)}\left(\theta_{1}, \theta_{2}, \ldots, \theta_{f}\right) \\
& =\frac{1}{c}\left(\bigoplus_{t=1}^{\otimes}\left(\begin{array}{c}
\left.\oplus_{q=1}^{b}\left(\omega_{l_{q}} \theta_{l_{q}}\right)\right)^{\frac{1}{c_{o_{t}}}} \\
1 \leqslant l_{1}<\ldots \\
<l_{b} \leqslant o_{t}
\end{array}\right)\right)
\end{aligned}
$$

where $\left(l_{1}, l_{2}, \ldots, l_{b}\right)$ contains all the b-tuple combination of $\left(1,2, \ldots, o_{t}\right)$ and $C_{o_{t}}^{b}=\frac{o_{t} !}{b !\left(o_{t}-b\right) !}$.

Theorem 6 For given IFNs $\theta_{l}=\left(\rho_{l}, \tau_{l}\right)(l=1,2, \ldots, f)$, the weight of IFN $\theta_{l}(l=1,2, \ldots, f)$ is $\omega_{b}$, meeting $\omega_{l} \in[0,1]$ and $\sum_{l=1}^{f} \omega_{l}=1$. The aggregated result of formula $(9)$ is too an IFN, listed:

$$
\begin{aligned}
& \operatorname{WIFPDMSM}^{(b)}\left(\theta_{1}, \theta_{2}, \ldots, \theta_{f}\right) \\
& =\left(1-\left(\prod_{t=1}^{c}\left(1-\prod_{\substack{1 \leqslant l_{1}<\ldots \\
<l_{b} \leqslant o_{t}}}\left(1-\prod_{q=1}^{b}\left(1-\rho_{l_{q}}\right)^{\omega_{l_{q}}}\right)^{\frac{1}{c_{o_{t}}}}\right)^{\frac{1}{b}}\right),\left(\prod_{t=1}^{c}\left(1-\prod_{\substack{\frac{1}{c} \\
1 \leqslant l_{1} \leqslant \ldots \\
<l_{b} \leqslant o_{t}}}\left(1-\prod_{q=1}^{b} \tau_{l_{q}}^{\omega_{l_{q}}}\right)^{\frac{1}{c_{o_{t}}}}\right)^{\frac{1}{b}}\right)\right) .
\end{aligned}
$$

Table 1 Decision matrix $\Lambda$ given by the decision maker

\begin{tabular}{lllllll}
\hline & $Z_{1}$ & $Z_{2}$ & $Z_{3}$ & $Z_{4}$ & $Z_{5}$ & $Z_{6}$ \\
\hline$Y_{1}$ & $(0.5,0.3)$ & $(0.6,0.3)$ & $(0.6,0.2)$ & $(0.4,0.4)$ & $(0.6,0.3)$ & $(0.4,0.3)$ \\
$Y_{2}$ & $(0.6,0.3)$ & $(0.3,0.4)$ & $(0.7,0.2)$ & $(0.6,0.3)$ & $(0.5,0.2)$ & $(0.6,0.4)$ \\
$Y_{3}$ & $(0.6,0.3)$ & $(0.5,0.2)$ & $(0.4,0.4)$ & $(0.5,0.3)$ & $(0.4,0.2)$ & $(0.3,0.3)$ \\
$Y_{4}$ & $(0.6,0.2)$ & $(0.7,0.3)$ & $(0.6,0.2)$ & $(0.6,0.3)$ & $(0.8,0.2)$ & $(0.7,0.2)$ \\
\hline
\end{tabular}


Table 2 Ranking results by our developed method for parameter

\begin{tabular}{lll}
\hline Parameter $b, c$ & Score value $S\left(\ddot{\theta}_{l}\right)(l=1,2,3,4)$ & Sorting \\
\hline$b=1, c=2$ & $S\left(\ddot{\theta}_{1}\right)=-0.706, S\left(\ddot{\theta}_{2}\right)=-0.707, \quad Y_{4}>Y_{1}>Y_{2}>Y_{3}$ \\
& $S\left(\ddot{\theta}_{3}\right)=-0.727, S\left(\ddot{\theta}_{4}\right)=-0.625$ & \\
$b=2, c=2$ & $S\left(\ddot{\theta}_{1}\right)=-0.697, S\left(\ddot{\theta}_{2}\right)=-0.692$, & $Y_{4}>Y_{2}>Y_{1}>Y_{3}$ \\
& $S\left(\ddot{\theta}_{3}\right)=-0.717, S\left(\ddot{\theta}_{4}\right)=-0.616$ & \\
$b=3, c=2$ & $S\left(\ddot{\theta}_{1}\right)=-0.694, S\left(\ddot{\theta}_{2}\right)=-0.687, \quad Y_{4}>Y_{2}>Y_{1}>Y_{3}$ \\
& $S\left(\ddot{\theta}_{3}\right)=-0.712, S\left(\ddot{\theta}_{4}\right)=-0.613$ \\
$b=1, c=1$ & $S\left(\ddot{\theta}_{1}\right)=-0.720, S\left(\ddot{\theta}_{2}\right)=-0.710, \quad Y_{4}>Y_{2}>Y_{1}>Y_{3}$ \\
& $S\left(\ddot{\theta}_{3}\right)=-0.730, S\left(\ddot{\theta}_{4}\right)=-0.635$ \\
$b=2, c=1$ & $S\left(\ddot{\theta}_{1}\right)=-0.704, S\left(\ddot{\theta}_{2}\right)=-0.697, \quad Y_{4}>Y_{2}>Y_{1}>Y_{3}$ \\
& $S\left(\ddot{\theta}_{3}\right)=-0.720, S\left(\ddot{\theta}_{4}\right)=-0.622$ \\
$b=3, c=1$ & $S\left(\ddot{\theta}_{1}\right)=-0.070, S\left(\ddot{\theta}_{2}\right)=-0.053, \quad Y_{4}>Y_{2}>Y_{1}>Y_{3}$ \\
& $S\left(\ddot{\theta}_{3}\right)=-0.093, S\left(\ddot{\theta}_{4}\right)=-0.050$
\end{tabular}

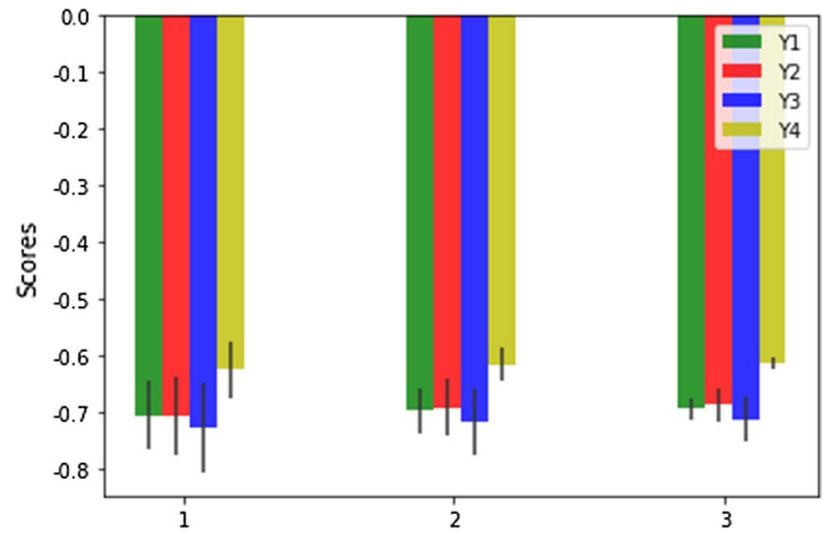

Fig. 1 Ranking results by our developed method for $c=2, b=1,2,3$

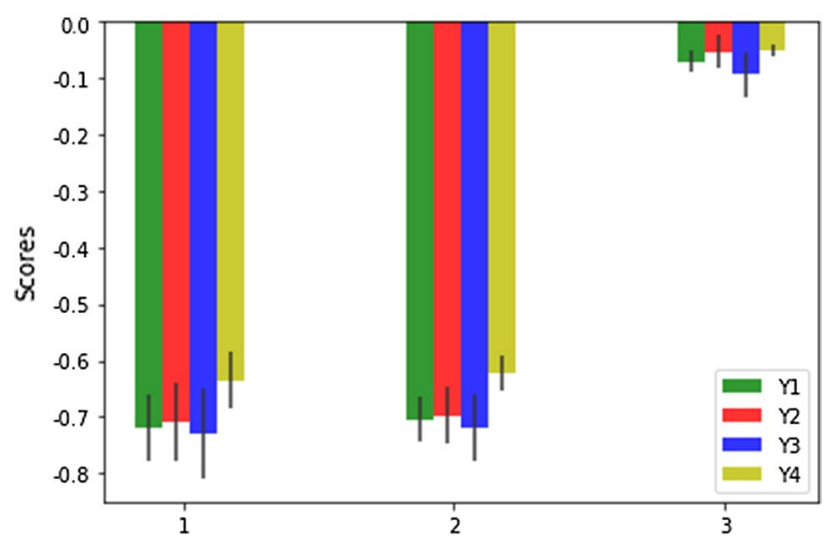

Fig. 2 Ranking results by our developed method for $c=1, b=1,2,3$
Likewise, we can analyze the WIFPDMSM operator has some desirable properties.

Proposition 8 (Commutativity) For given IFNs $\theta_{l}=\left(\rho_{l}, \tau_{l}\right)$ and $\ddot{\theta}_{l}=\left(\ddot{\rho}_{l}, \ddot{\tau}_{l}\right)(l=1,2, \ldots, f)$. Suppose $\ddot{\theta}_{l}=\left(\ddot{\rho}_{l}, \ddot{\tau}_{l}\right)$ is any permutation of $\theta_{l}=\left(\rho_{l}, \tau_{l}\right)$ for all $l$, then

$$
\begin{aligned}
& \operatorname{WIFPDMSM}^{(b)}\left(\theta_{1}, \theta_{2}, \ldots, \theta_{f}\right) \\
& \quad=\operatorname{WIFPDMSM}^{(b)}\left(\ddot{\theta}_{1}, \ddot{\theta}_{2}, \ldots, \ddot{\theta}_{f}\right) .
\end{aligned}
$$

Proposition 9 (Monotonicity) For given IFNs $\theta_{l}=\left(\rho_{l}, \tau_{l}\right)$ and $\ddot{\theta}_{l}=\left(\ddot{\rho}_{l}, \ddot{\tau}_{l}\right)(l=1,2, \ldots, f)$, satisfying the condition $\rho_{l} \geqslant \ddot{\rho}_{l}, \tau_{l} \leqslant \ddot{\tau}_{l}$, then

$$
\begin{aligned}
& \operatorname{WIFPDMSM}^{(b)}\left(\theta_{1}, \theta_{2}, \ldots, \theta_{f}\right) \\
& \quad \geqslant \operatorname{WIFPDMSM}^{(b)}\left(\ddot{\theta}_{1}, \ddot{\theta}_{2}, \ldots, \ddot{\theta}_{f}\right) .
\end{aligned}
$$

Furthermore, we discuss the effect of parameter $b$ about the WIFPDMSM operator and particular case.

Theorem 7 Let $\theta_{l}=\left(\rho_{l}, \tau_{l}\right)(l=1,2, \ldots, f)$ be a collection of IFNs, $b=1,2, \ldots, \min _{t}\left\{o_{t}\right\}$. The WIFPDMSM operator monotonically increases with respect to the $b$.

Theorem 8 Let $\theta_{l}=\left(\rho_{l}, \tau_{l}\right)(l=1,2, \ldots, f)$ be a set of IFNs, $b=1,2, \ldots, \min _{t}\left\{o_{t}\right\}$. Then

$$
\begin{aligned}
\min & \left\{\operatorname{WIFPDMSM}^{(b)}\left(\theta_{1}, \theta_{2}, \ldots, \theta_{f}\right)\right\} \\
= & \operatorname{WIFPDMSM}^{(1)}\left(\theta_{1}, \theta_{2}, \ldots, \theta_{f}\right) \\
= & \left(1-\left(\prod_{t=1}^{c}\left(1-\prod_{l_{q}=1}^{o_{t}}\left(1-\left(1-\rho_{l_{q}}\right)^{\omega_{l_{q}}}\right)^{\frac{1}{o_{t}}}\right)\right)^{\frac{1}{c}},\right. \\
& \left.\left(\prod_{t=1}^{c}\left(1-\prod_{l_{q}=1}^{o_{t}}\left(1-\tau_{l_{q}}^{\omega_{l_{q}}}\right)^{\frac{1}{o_{t}}}\right)\right)^{\frac{1}{c}}\right)
\end{aligned}
$$

and

$$
\begin{aligned}
\max & \left\{\operatorname{WIFPDMSM}^{(b)}\left(\theta_{1}, \theta_{2}, \ldots, \theta_{f}\right)\right\} \\
& =\operatorname{WIFPDMSM}_{t}^{\left(\min _{t}\left\{o_{t}\right\}\right)}\left(\theta_{1}, \theta_{2}, \ldots, \theta_{f}\right) .
\end{aligned}
$$

when $c=1$, we can get 
Table 3 New decision matrix $\Lambda^{\prime}$ got by the decision maker

\begin{tabular}{lllllll}
\hline & $Z_{1}$ & $Z_{2}$ & $Z_{3}$ & $Z_{4}$ & $Z_{5}$ & $Z_{6}$ \\
\hline$Y_{1}$ & $(0.5,0.3)$ & $(0.6,0.3)$ & $(0.6,0.2)$ & $(0.4,0.4)$ & $(0.6,0.3)$ & $(0.4,0.3)$ \\
$Y_{2}$ & $(0.6,0.3)$ & $(0.3,0.4)$ & $(0.7,0.2)$ & $(0.6,0.3)$ & $(0.5,0.2)$ & $(0.6,0.4)$ \\
$Y_{3}^{\prime}$ & $(0.5,0.4)$ & $(0.3,0.3)$ & $(0.3,0.5)$ & $(0.4,0.4)$ & $(0.3,0.3)$ & $(0.2,0.5)$ \\
$Y_{4}$ & $(0.6,0.2)$ & $(0.7,0.3)$ & $(0.6,0.2)$ & $(0.6,0.3)$ & $(0.8,0.2)$ & $(0.7,0.2)$ \\
\hline
\end{tabular}

Table 4 Sorting of diverse methodologies of Example 2

\begin{tabular}{lll}
\hline Methodologies & Score values & Sorting \\
\hline Xu and Yager [3] & $S\left(\ddot{\theta}_{1}\right)=0.220, S\left(\ddot{\theta}_{2}\right)=0.214$, & $Y_{4}>Y_{1}>Y_{2}>Y_{3}$ \\
& $S\left(\ddot{\theta}_{3}\right)=0.144, S\left(\ddot{\theta}_{4}\right)=0.425$ & \\
Qin and Liu $(b=2)[19]$ & $S\left(\ddot{\theta}_{1}\right)=-0.701, S\left(\ddot{\theta}_{2}\right)=-0.694$, & $Y_{4}>Y_{2}>Y_{1}>Y_{3}$ \\
& $S\left(\ddot{\theta}_{3}\right)=-0.718, S\left(\ddot{\theta}_{4}\right)=-0.619$ & \\
Xia et al. $(q=p=1)[25]$ & $S\left(\ddot{\theta}_{1}\right)=0.887, S\left(\ddot{\theta}_{2}\right)=0.890$, & $Y_{4}>Y_{2}>Y_{1}>Y_{3}$ \\
& $S\left(\ddot{\theta}_{3}\right)=0.873, S\left(\ddot{\theta}_{4}\right)=0.923$ & \\
Shi and Xiao* $(b=2)[40]$ & $S\left(\ddot{\theta}_{1}\right)=0.222, S\left(\ddot{\theta}_{2}\right)=0.248$, & $Y_{4}>Y_{2}>Y_{1}>Y_{3}$ \\
& $S\left(\ddot{\theta}_{3}\right)=0.164, S\left(\ddot{\theta}_{4}\right)=0.433$ & \\
Shi and Xiao** $(b=2)[40]$ & $S\left(\ddot{\theta}_{1}\right)=0.219, S\left(\ddot{\theta}_{2}\right)=0.246$, & $Y_{4}>Y_{2}>Y_{1}>Y_{3}$ \\
& $S\left(\ddot{\theta}_{3}\right)=0.163, S\left(\ddot{\theta}_{4}\right)=0.434$ & \\
The developed approach $(b=2, c=2)$ & $S\left(\ddot{\theta}_{1}\right)=-0.697, S\left(\ddot{\theta}_{2}\right)=-0.692$, & $Y_{4}>Y_{2}>Y_{1}>Y_{3}$ \\
& $S\left(\ddot{\theta}_{3}\right)=-0.717, S\left(\ddot{\theta}_{4}\right)=-0.616$ & \\
\hline
\end{tabular}

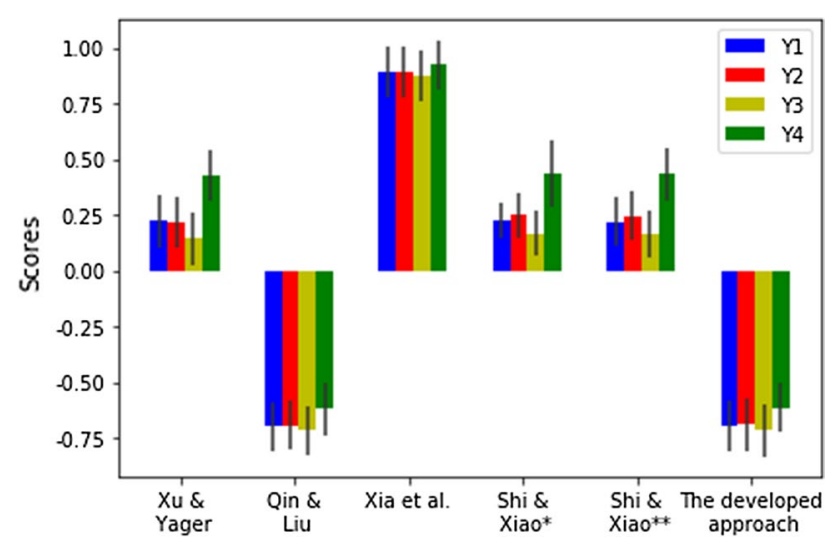

Fig. 3 Sorting of diverse methodologies of Example 2

$$
\begin{aligned}
& \operatorname{WIFPDMSM}^{(b)}\left(\theta_{1}, \theta_{2}, \ldots, \theta_{f}\right) \\
& =\left(1-\left(1-\prod_{\substack{1 \leqslant l_{1}<\ldots \\
<l_{b} \leqslant f}}\left(1-\prod_{q=1}^{b}\left(1-\rho_{l_{q}}\right)^{\omega_{l_{q}}}\right)^{\frac{1}{c_{f}^{b}}}\right)^{\frac{1}{b}},\right. \\
& \left.\left(1-\prod_{1 \leqslant l_{1}<\cdots<l_{b} \leqslant f}\left(1-\prod_{q=1}^{b} \tau_{l_{q}}^{\omega_{l_{q}}}\right)^{\frac{1}{c_{f}^{b}}}\right)^{\frac{1}{b}}\right) .
\end{aligned}
$$

\section{MADM Approach Based on}

Following, we will apply an established MADM methodology to solve real decision-making problem. Suppose $Y=\left\{Y_{1}, Y_{2}, \ldots, Y_{n}\right\}$ be a group of alternatives, $Z=\left\{Z_{1}, Z_{2}, \ldots, Z_{f}\right\}$ be a set of attributes, let $\omega=\left(\omega_{1}, \omega_{2}, \ldots, \omega_{f}\right)$ is weight vector of attributes and $\sum_{l=1}^{f} \omega_{l}=1,0 \leqslant \omega_{l} \leqslant 1(l=1,2, \ldots, f)$. Decision maker can use the IFNs to evaluate the alternative $Y_{l}$ with respect to attribute $Z_{q}$ and represent it as $\theta_{l_{q}}=\left(\rho_{l_{q}}, \tau_{l_{q}}\right)$, where $\rho_{l_{q}}, \tau_{l_{q}} \in[0,1]$ and $0 \leqslant \rho_{l_{q}}+\tau_{l_{q}} \leqslant 1$, we can get a decision matrix $\Lambda=\left[\theta_{l_{q}}\right]_{n \times f}, 1 \leqslant l \leqslant n, 1 \leqslant q \leqslant f$. Suppose that $Z=\left\{Z_{1}, Z_{2}, \ldots, Z_{f}\right\}$ is partitioned into $c$ distinct groups $P_{1}, P_{2}, \ldots, P_{c}$, there has relationship between attributes of the identical group and does not have relationship between attributes of disparate groups. The steps of the propounded method are listed:

Step 1 In DMPs, attributes can be cut into two types, one is benefit attribute, and the other is cost attribute. In order to eliminate the effect of different attribute types, we can transform the decision matrix $\Lambda=\left[\theta_{l_{q}}\right]_{n \times f}$ into the normalization matrix $\ddot{\Lambda}=\left[\ddot{\theta}_{l_{q}}\right]_{n \times f}, 1 \leqslant l \leqslant n, 1 \leqslant q \leqslant f$. We use the following formula to obtain the normalization matrix $\ddot{\Lambda}=\left[\ddot{\theta}_{l_{q}}\right]_{n \times f}$ $\ddot{\theta}_{l_{q}}=\left(\ddot{\rho}_{l_{q}}, \ddot{\tau}_{l_{q}}\right)= \begin{cases}\left(\rho_{l_{q}}, \tau_{l_{q}}\right), & \text { for the benefit attributes } \\ \left(\tau_{l_{q}}, \rho_{l_{q}}\right), & \text { for the cost attributes }\end{cases}$ 
where $1 \leqslant l \leqslant n, 1 \leqslant q \leqslant f$.

Step 2 Use the proposed WIFPDMSM operator to get

$\ddot{\theta}_{l}=\left(\ddot{\rho}_{l}, \ddot{\tau}_{l}\right)=\operatorname{WIFPDMSM}^{(b)}\left(\ddot{\theta}_{l_{1}}, \ddot{\theta}_{l_{2}}, \ldots, \ddot{\theta}_{l_{f}}\right)$

where $\ddot{\theta}_{l}$ is the preference value of alternative $Y_{l}$, where $l=1,2, \ldots, n$.

Step 3 Compute values $S\left(\ddot{\theta}_{l}\right)$ and $H\left(\ddot{\theta}_{l}\right)$ of the aggregated result $\ddot{\theta}_{l}(l=1,2, \ldots, n)$.

Step 4 According to the comparative way, making a comparing between the computed values $S\left(\ddot{\theta}_{1}\right), S\left(\ddot{\theta}_{2}\right), \ldots, S\left(\ddot{\theta}_{n}\right)$ and accuracy values $H\left(\ddot{\theta}_{1}\right), H\left(\ddot{\theta}_{2}\right), \ldots, H\left(\ddot{\theta}_{n}\right)$, then ranking all alternatives to choose the greatest one(s).

\subsection{Problem Description}

Example 2 In the north, there are often droughts in summer, which has some effect on the crops and causes the loss of many farmers. In order to cut the loss of farmers as soon as possible, the local government asked a company to build a dam. After the company goes through many surveys, there are four possible locations $Y_{1}, Y_{2}, Y_{3}, Y_{4}$ and decision maker assesses the four possible locations from six attributes, i.e., the cost of location $\left(Z_{1}\right)$, the size of location $\left(Z_{2}\right)$, the surrounding environment $\left(Z_{3}\right)$, the labor cost $\left(Z_{4}\right)$, the material cost $\left(Z_{5}\right)$ and the transportation condition $\left(Z_{6}\right)$. Let the weights of attributes $Z_{1}, Z_{2}, Z_{3}, Z_{4}, Z_{5}$ and $Z_{6}$ be $0.2,0.2,0.2,0.1,0.1$ and 0.2 , respectively, the weight vector is $(0.2,0.2,0.2,0.1,0.1,0.2)^{T}$. Furthermore, suppose that the attributes $Z_{1}, Z_{2}, Z_{3}, Z_{4}, Z_{5}$ and $Z_{6}$ are cut into two groups $P_{1}$ and $P_{2}$, where $P_{1}=\left\{Z_{1}, Z_{4}, Z_{5}\right\}$ and $P_{2}=\left\{Z_{2}, Z_{3}, Z_{6}\right\}$. The decision maker evaluates the location $Y_{l}(l=1,2,3,4)$ with respect to the attribute $Z_{q}(q=1,2,3,4,5,6)$ by the IFNs. Therefore, the decision matrix $\Lambda=\left[\theta_{l_{q}}\right]_{4 \times 6}$ can be obtained, where $\theta_{l_{q}}=\left(\rho_{l_{q}}, \tau_{l_{q}}\right), 1 \leqslant l \leqslant 4,1 \leqslant q \leqslant 6$, which is shown as follows (Table 1).

\subsection{Decision-Making Process About the Designed Method}

Step 1Standardize the matrix $\Lambda$. Because total attributes are beneficial, standardization does not need.

Step 2 Aggregate all performance value $\ddot{\theta}_{l_{q}}(1 \leqslant l \leqslant 4,1 \leqslant q \leqslant 6)$. And founded on Eq. (11), we get the aggregated result $\ddot{\theta}_{l}$ for each alternative $Y_{l}(l=1,2,3,4)$. (Without loss of generality, here we take $b=2, c=2$ )

$\ddot{\theta}_{1}=(0.116,0.813)$,

$\ddot{\theta}_{2}=(0.127,0.820)$,

$\ddot{\theta}_{3}=(0.095,0.811)$,

$\ddot{\theta}_{4}=(0.167,0.783)$.

Step 3 Compute value $S\left(\ddot{\theta}_{l}\right)$ and $H\left(\ddot{\theta}_{l}\right)$ of the overall preference value $\ddot{\theta}_{l}(l=1,2,3,4)$, presented below:

$S\left(\ddot{\theta}_{1}\right)=-0.697, S\left(\ddot{\theta}_{2}\right)=-0.692$,

$S\left(\ddot{\theta}_{3}\right)=-0.717, S\left(\ddot{\theta}_{4}\right)=-0.616$,

$H\left(\ddot{\theta}_{1}\right)=0.929, H\left(\ddot{\theta}_{2}\right)=0.947$,

$H\left(\ddot{\theta}_{3}\right)=0.906, H\left(\ddot{\theta}_{4}\right)=0.950$.

Step 4 In accordance with the Step 3, the sorting result is $Y_{4}>Y_{2}>Y_{1}>Y_{3}$. Therefore, the greatest alternative is $Y_{4}$.

Furthermore, we can use the parameter $b$ to describe the preference of decision maker in real situations. So, decision maker need to choose the suitable value based on preference. The decision maker is either optimistic or pessimistic when values of the parameter $b$ are different [44]. We discuss the effect of the different value of the parameter $b$ about the developed method. In Table 2 and Figs. 1 and 2, we can obtain the results by the propounded method.

From Table 2 and Figs. 1 and 2., we can get: with the parameter $b$ changing based on the subjective preference of decision maker, the sorting results are slightly different,

Table 5 The characteristics of the distinct approaches

\begin{tabular}{lllll}
\hline Approaches & $\begin{array}{l}\text { Depict fuzzy informa- } \\
\text { tion using NMD }\end{array}$ & $\begin{array}{l}\text { Capture relationship for each two } \\
\text { attributes of the identical group }\end{array}$ & $\begin{array}{l}\text { Capture relationship for attrib- } \\
\text { utes of the identical group }\end{array}$ & $\begin{array}{l}\text { Consider the } \\
\text { parts for the } \\
\text { attributes }\end{array}$ \\
\hline Xu and Yager [3] & Yes & No & No & No \\
Qin and Liu [19] & Yes & Yes & Yes & No \\
Xia et al. [25] & Yes & Yes & No & No \\
Shi and Xiao* [40] & Yes & Yes & Yes & No \\
Shi and Xiao** [40] & Yes & Yes & Yes & Yes \\
The developed approach & Yes & Yes & &
\end{tabular}


which denotes that the WIFPDMSM operator can reflect the preference of decision maker, but the optimal alternative is all $Y_{4}$. And we can get score value increases based on the developed operator when the value of $b$ increases and the value of $c$ is fixed.

\subsection{Validation Test}

We examine the effectiveness of the developed approach through Wang and Triantaphylou introduced three test criterion, the specific content of three test criterion is in [48].

Firstly, to verify the effectiveness of the developed methodology by Test Criterion 1 [48]. The new decision matrix is got by decision maker through replacing alternative $Y_{3}$ by a worse alternative $Y_{3}^{\prime}$, shown in Table 3 .

We use the established methodology to resolve the new decision-making problem. The outcome of each alternative is calculated as

$\ddot{\theta}_{1}=(0.116,0.813), \ddot{\theta}_{2}=(0.127,0.820)$,

$\ddot{\theta}_{3}=(0.064,0.860), \ddot{\theta}_{4}=(0.167,0.783)$.

So, the score value of each alternative is computed as

$S\left(\ddot{\theta}_{1}\right)=-0.697, S\left(\ddot{\theta}_{2}\right)=-0.692$,

$S\left(\ddot{\theta}_{3}\right)=-0.796, S\left(\ddot{\theta}_{4}\right)=-0.616$.

Founded on the above discussion, the sorting is $Y_{4}>Y_{2}>Y_{1}>Y_{3}$, the optimal alternative is still alternative $Y_{4}$ in the new decision-making problem, which is similar with the result obtained by the original problem. So the optimal alternative is not changed when the non-optimal alternative is replaced with another worse alternative.

Next, we examine the effectiveness of our presented methodology utilizing Test Criterion 2,3 [48]. Firstly, the initial decision issue decomposes as the three smaller problems $\left\{Y_{1}, Y_{2}, Y_{4}\right\},\left\{Y_{1}, Y_{3}, Y_{4}\right\},\left\{Y_{2}, Y_{3}, Y_{4}\right\}$. Then, we use the developed method in this paper to solve the three smaller problems, respectively, the corresponding sorting results are obtained as $Y_{4}>Y_{2}>Y_{1}, Y_{4}>Y_{1}>Y_{3}, Y_{4}>Y_{2}>Y_{3}$. Accordingly, the combination ranking is $Y_{4}>Y_{2}>Y_{1}>Y_{3}$, which is identical with sorting of the initial decision issue and meets the transitivity property. Thus, the proposed approach satisfies the Test criterion 2 and Test criterion 3 .

\subsection{Comparison Analysis}

In the following, in order to confirm the validity of the propounded methodology, we compare the presented methodology with other methods, such as Xu and Yager's MADM approach [3] using the IFWG operator, Qin and Liu's MADM methodology [19] utilizing the WIFMSM operator, Xia et al.'s MADM approach [25] using the WIFGBM operator, Shi and Xiao's MADM method [40] utilizing the IFRWDMSM and the IFRWMSM operator based on Example 2, and the result of each method is shown in Table 4 and Fig. 3 (* denotes the method [40] grounded on the IFRWDMSM operator, $* *$ denotes the method [40] based on the IFRWMSM operator). In Table 4 and Fig. 3., we can get that the decision outcomes through applying different approaches have subtle difference. But, the most satisfied option is all $Y_{4}$. Furthermore, we analyze why the developed method outperforms other methods, listed as follows:

(1) MADM methodology utilizes the WIFMSM operator propounded by Qin and Liu [19], MADM method utilizes the WIFGBM operator established by Xia et al. [25], MADM approach utilizes the IFRWDMSM and the IFRWMSM operator introduced by Shi and Xiao [40], but our developed method uses the propounded WIFPDMSM operator. From Table 4, we can see that the sortings of Qin and Liu's MADM methodology [19], Xia et al.'s MADM method [25] and Shi and Xiao's MADM approach [40] are same to the designed MADM method based on the WIFPDMSM operator, namely $Y_{4}>Y_{2}>Y_{1}>Y_{3}$. The proposed method considers the interrelationship betwixt attributes in the identical group; there are other methods capture correlation between attributes except the Xia et al.'s MADM method [25] which captures the correlation for any two attributes. In Example 2, attributes $Z_{1}, Z_{4}$ and $Z_{5}$ are related to each other and attributes $Z_{2}, Z_{3}$ and $Z_{6}$ are so too, but there is no interrelationship between the groups $\left\{Z_{1}, Z_{4}, Z_{5}\right\}$ and $\left\{Z_{2}, Z_{3}, Z_{6}\right\}$. Obviously, our method based on the introduced WIFPDMSM operator effectively settles Example 2, which views the relationship betwixt any two members of the identical set, it averts the influence of irrelevant members. Qin and Liu's MADM approach [19], Xia et al.'s MADM approach [25] and Shi and Xiao's MADM approach [40] consider correlation between attributes, but these attributes are independent each other, for example among the attributes $Z_{1}$ and $Z_{2}$, among the attributes $Z_{1}$ and $Z_{3}$, among the attributes $Z_{1}$ and $Z_{6}$, among the attributes $Z_{4}$ and $Z_{2}$, among the attributes $Z_{4}$ and $Z_{3}$, among the attributes $Z_{4}$ and $Z_{6}$, among the attributes $Z_{5}$ and $Z_{2}$, among the attributes $Z_{5}$ and $Z_{3}$, among the attributes $Z_{5}$ and $Z_{6}$. Thus, the developed methodology grounded on the WIFPDMSM operator contrasting with Qin and Liu's MADM methodology [19], Xia et al.'s MADM method [25] and Shi and Xiao's MADM method [40] is more general.

(2) The sorting of MADM approach [3] grounded on the IFWG operator introduced by $\mathrm{Xu}$ and Yager is slightly different from the sorting of the established method, 
because $\mathrm{Xu}$ and Yager's MADM approach uses ordinary weighted geometric operator, which does not view relationship for the attributes. In Example 2, attributes $Z_{1}, Z_{4}$ and $Z_{5}$ are relevant and attributes $Z_{2}, Z_{3}$ and $Z_{6}$ are relevant. Therefore, the developed method successfully settles Example 2.

(3) In real DMPs, some attributes may be independent each other. Thus, the developed method is more suitably used in practical MADM issues by contrasting with the methods $[19,25,40]$. And in real DMPs, relationship of attribute may be not ignored. Therefore, the designed method is more generally used in practical MADM issues by contrasting with the approach [3].

By previous analysis, the characteristics of the put forward methodology contrast with other methodologies, results are listed in Table 5 (* denotes the method [40] grounded on the IFRWDMSM operator, ** denotes the method [40] based on the IFRWMSM operator). From it, we can obtain: (1) Xu and Yager's MADM approach [3] does not consider correlation between attributes, Xia et al.'s MADM approach [25] capture relationship between any two attributes, Qin and Liu's MADM methodology [19], Shi and Xiao's MADM approach [40] and the propounded method consider interrelationship for the attributes; (2) the developed MADM method partitions overall attributes into some parts and the attributes are interrelated in the same part, but the attributes are not interrelated in different parts, while Qin and Liu's MADM methodology [19], Xia et al.'s MADM methodology [25] and Shi and Xiao's MADM approach [40] don't split attributes into several portions; (3) the proposed approach and Xia et al.'s MADM approach [25] consider the interrelation between attributes. Our proposed MADM method views interrelationship betwixt members of the identical parts, but Xia et al.'s MADM methodology [25] just finds relation betwixt any two members; (4) our introduced approach can surmount the disadvantage of Qin and Liu's MADM methodology [19], Xia et al.'s MADM approach [25] and Shi and Xiao's MADM approach [40], where the four approaches do not cut the attributes into some partitions, and $\mathrm{Xu}$ and Yager's MADM approach [3] does not capture the interrelationship between attributes. In conclusion, the propounded methodology is more ordinary and appropriate than other methods $[3,19,25,40]$.

\section{Conclusion and Future Studies}

The DMSM operator considers that attributes are existing multiple relation. We easily find that the generalizations of the DMSM just only consider the case that attributes are relevant. But, in real DMPs, there may be not relationship between attributes So this paper has put forward the PDMSM operator. The purposed PDMSM operator has been expanded to the IFPDMSM and the WIFPDMSM operator for intuitionistic fuzzy environment. A few features and particular instances of the established operators have been analyzed. In accordance with the propounded WIFPDMSM operator, a new MADM approach has been designed for intuitionistic fuzzy environment. The established method breaks attributes into some portions, considering attributes of the identical portion are interrelated, while attributes of diverse portions are independent each other. We have applied an example to contrast our established MADM approach with the ones of $[3,19,25,40]$, comparative results show our introduced MADM methodology is more effective than Qin and Liu's MADM methodology [19], Xia et al.'s MADM approach [25], Shi and Xiao's MADM approach [40], and Xu and Yager's MADM approach [3] in which does not capture the interrelationship between attributes to deal with MADM problems with intuitionistic fuzzy environment. In our future study, the presented operators will be utilized to fuzzy linguistic, which is admissive to utilize multiple linguistic fuzzy sets in fuzzy linguistic, it has been wide utilized in DMPs because which allows decision makers to use linguistic fuzzy set to voice their preferences. And the introduced operators can be used in proportional hesitant fuzzy 2-tuple linguistic term set [49] to deal with DMPs. The study is in intuitionistic fuzzy environment, spherical fuzzy sets, Pythagorean fuzzy sets and q-rung orthopair fuzzy sets are extension of IFS, and the introduced operator PDMSM can be used for spherical fuzzy sets $[50,51]$ which introduced operations of spherical fuzzy sets and Pythagorean fuzzy sets [52] which introduced operators and q-rung orthopair fuzzy sets $[53,54]$ which introduced a new type and ranking of q-rung orthopair fuzzy sets to solve DMPS. The established operator PDMSM can combine with TOPSIS method $[55,56]$ which established similarity and distance of Complex Interval-Valued q-Rung Orthopair Fuzzy Sets and consider the unknown weights of the attributes to solve DMPS. These are future works.

Acknowledgements This work was supported by Sichuan Province Youth Science and Technology Innovation Team under Grant 2019JDTD0015, Application Basic Research Plan Project of Sichuan Province under Grant 2021JY0108, Scientific Research Project of Neijiang Normal University under Grant 18TD08, Scientific Research Project of Neijiang Normal University under Grant 2019YB11.

\section{Declarations}

Conflict of interest There is no any conflicts of interest among all authors.

Open Access This article is licensed under a Creative Commons Attribution 4.0 International License, which permits use, sharing, 
adaptation, distribution and reproduction in any medium or format, as long as you give appropriate credit to the original author(s) and the source, provide a link to the Creative Commons licence, and indicate if changes were made. The images or other third party material in this article are included in the article's Creative Commons licence, unless indicated otherwise in a credit line to the material. If material is not included in the article's Creative Commons licence and your intended use is not permitted by statutory regulation or exceeds the permitted use, you will need to obtain permission directly from the copyright holder. To view a copy of this licence, visit http://creativecommons. org/licenses/by/4.0/.

\section{References}

1. Zadeh, L.A.: Fuzzy sets. Inf. Control 8, 338-353 (1965)

2. Atanassov, K.T.: Intuitionistic fuzzy sets. Fuzzy Sets Syst. 20, 87-96 (1986)

3. Xu, Z.S., Yager, R.R.: Some geometric aggregation operators based on intuitionistic fuzzy sets. Int. J. Gen. Syst. 35, 417-433 (2006)

4. Garg, H., Kumar, K.: Improved possibility degree method for ranking intuitionistic fuzzy numbers and their application in multiattribute decision making. Granul. Comput. 4, 237-247 (2019)

5. Joshi, R.: A new multi-criteria decision-making method based on intuitionistic fuzzy information and its application to fault detection in a machine. J. Ambient. Intell. Humaniz. Comput. 11, 739-753 (2020)

6. Liu, P.D., Wang, P.: Some improved linguistic intuitionistic fuzzy aggregation operators and their applications to multiple-attribute decision making. Int. J. Inf. Technol. Decis. Mak. 16, 817-850 (2017)

7. Peng, H.G., Wang, J.Q., Cheng, P.F.: A linguistic intuitionistic multi-criteria decision making method based on the Frank Heronian mean operator and its application in evaluating coal mine safety. Int. J. Mach. Learn. Cybern. 9, 1053-1068 (2017)

8. Qiyas, M., Abdullah, S., Liu, Y., Naeem, M.: Multi-criteria decision support systems based on linguistic intuitionistic cubic fuzzy aggregation operators. J. Ambient. Intell. Humaniz. Comput. 12, 8285-8303 (2021)

9. Liu, P.D., Chen, S.M.: Multiattribute group decision making based on intuitionistic 2-tuple linguistic information. Inf. Sci. 430, 599 619 (2018)

10. Chen, Z.C., Liu, P.H., Pei, Z.: An approach to multiple attribute group decision making based on linguistic intuitionistic fuzzy numbers. Int. J. Comput. Intell. Syst. 8, 747-760 (2015)

11. Teng, F., Liu, P.D.: Multiple-attribute group decision-making method based on the linguistic intuitionistic fuzzy density hybrid weighted averaging operator. Int. J. Fuzzy Syst. 21, 213-231 (2018)

12. Zhang, Y.J., Hu, S.H., Zhou, W.: Multiple attribute group decision making using J-divergence and evidential reasoning theory under intuitionistic fuzzy environment. Neural Comput. Appl. 32, 6311-6326 (2020)

13. Molodtsov, D.: Soft set theory-first results. Comput. Math. Appl. 37, 19-31 (1999)

14. Lee, K.M.: Bipolar valued fuzzy sets and their operations. In: Proceedings of International Conference on Intelligent Technologies, Bangkok, Thailand, pp. 307-312 (2000)

15. Mahmood, T.: A novel approach towards bipolar soft sets and their applications. J. Math. (2020). https://doi.org/10.1155/2020/46908 08
16. Mahmood, T., Rehman, U.U., Ali, Z.: A novel complex fuzzy $\mathrm{N}$-soft sets and their decision-making algorithm. Complex Intell. Syst. (2021). https://doi.org/10.1007/s40747-021-00373-2

17. Zhao, M.W., Wei, G.W., Wei, C., Guo, Y.F.: CPT-TODIM method for bipolar fuzzy multi-attribute group decision making and its application to network security service provider selection. Int. J. Intell. Syst. 36, 1943-1969 (2021)

18. Jana, C., Pal, M., Wang, J.Q.: Bipolar fuzzy Dombi aggregation operators and its application in multiple-attribute decision-making process. J. Ambient. Intell. Humaniz. Comput. 10, 3533-3549 (2019)

19. Qin, J.D., Liu, X.W.: An approach to intuitionistic fuzzy multiple attribute decision making based on Maclaurin symmetric mean operators. J. Intell. Fuzzy Syst. 27, 2177-2190 (2014)

20. Tao, Z.F., Han, B., Chen, H.Y.: On intuitionistic fuzzy copula aggregation operators in multiple-attribute decision making. Cogn. Comput. 10, 610-624 (2018)

21. Zhao, X.F., Wei, G.W.: Some intuitionistic fuzzy Einstein hybrid aggregation operators and their application to multiple attribute decision making. Knowl. Based Syst. 37, 472-479 (2013)

22. Xu, Z.S.: Intuitionistic fuzzy aggregation operators. IEEE Trans. Fuzzy Syst. 15, 1179-1187 (2007)

23. Xu, Z.S., Yager, R.R.: Intuitionistic fuzzy Bonferroni means. IEEE Trans. Fuzzy Syst. Man Cybern. Part B (Cybern.) 41, 568-578 (2011)

24. Bonferroni, C.: Sulle medie multiple di potenze. Bolletino Matematica Italiana 5, 267-270 (1950)

25. Xia, M.M., Xu, Z.S., Zhu, B.: Geometric Bonferroni means with their application in multi-criteria decision making. Knowl. Based Syst. 40, 88-100 (2013)

26. Dutta, B., Labella, A., Rodrguez, R.M., Martnez, L.: Aggregating interrelated attributes in multi-attribute decision making with ELICIT information based on Bonferroni mean and its variants. Int. J. Comput. Intell. Syst. 12, 1179-1196 (2019)

27. Sykora, S. (ed.): Mathematical Means and Averages: Generalized Heronian Means. Stan's Library. (2009). https://doi.org/10.3247/ SL3Math09.002

28. Liu, P.D., Liu, J.L., Merigo, J.M.: Partitioned Heronian means based on linguistic intuitionistic fuzzy numbers for dealing with multi-attribute group decision making. Appl. Soft Comput. 62, 395-422 (2018)

29. Mo, J.M., Huang, H.L.: Archimedean geometric Heronian mean aggregation operators based on dual hesitant fuzzy set and their application to multiple attribute decision making. Soft Comput. 24, 14721-14733 (2020)

30. Maclaurin, C.: A second letter to Martin Folkes, Esq concerning the roots of equations, with demonstration of other rules of algebra. Philos. Trans. R. Soc. Lond. Ser. A 36, 59-96 (1729)

31. Detemple, D., Robertson, J.: On generalized symmetric means of two variables. Univ. Beograd. Publ. Elektrotehn. Fak. Ser. Mat. Fiz. 634, 236-238 (1979)

32. Fahmi, A., Yaqoob, N., Chammam, W.: Maclaurin symmetric mean aggregation operators based on cubic Pythagorean linguistic fuzzy number. J. Ambient. Intell. Humaniz. Comput. 12(2), 1925-1942 (2021)

33. Liu, P.D., Li, Y., Zhang, M.C.: Some Maclaurin symmetric mean aggregation operators based on two-dimensional uncertain linguistic information and their application to decision making. Neural Comput. Appl. 31, 4305-4318 (2019)

34. Liu, P.D., You, X.L.: Linguistic neutrosophic partitioned Maclaurin symmetric mean operators based on clustering algorithm and their application to multi-criteria group decision-making. Artif. Intell. Rev. 53, 2131-2170 (2020) 
35. Sahin, R., Kucuk, G.D.: A novel group decision-making method based on linguistic neutrosophic Maclaurin symmetric mean. Cogn. Comput. 12, 699-717 (2020)

36. Liu, P.D., Yang, H.Y.: Three-way decisions with intuitionistic uncertain linguistic decision-theoretic rough sets based on generalized Maclaurin symmetric mean operators. Int. J. Fuzzy Syst. 22, 653-667 (2020)

37. Wang, J., Wei, G.W., Wang, R., Alsaadi, F.E., Hayat, T., Wei, C., Zhang, Y., Wu, J.: Some q-rung interval-valued orthopair fuzzy Maclaurin symmetric mean operators and their applications to multiple attribute group decision making. Int. J. Intell. Syst. 34, 2769-2806 (2019)

38. Garg, H., Arora, R.: Maclaurin symmetric mean aggregation operators based on t-norm operations for the dual hesitant fuzzy soft set. J. Ambient. Intell. Humaniz. Comput. 11, 375-410 (2020)

39. Qin, J.D., Liu, X.W.: Approaches to uncertain linguistic multiple attribute decision making based on dual Maclaurin symmetric mean. J. Intell. Fuzzy Syst. 29, 171-186 (2015)

40. Shi, M.H., Xiao, Q.X.: Intuitionistic fuzzy reducible weighted Maclaurin symmetric means and their application in multiple attribute decision making. Soft Comput. 23, 10029-10043 (2019)

41. Darko, A.P., Liang, D.C.: An extended COPRAS method for multiattribute group decision making based on dual hesitant fuzzy Maclaurin symmetric mean. Int. J. Intell. Syst. 35, 1021-1068 (2020)

42. Wang, J., Wei, G.W., Gao, H.: Approaches to multiple attribute decision making with interval-valued 2-tuple linguistic Pythagorean fuzzy information. Mathematics 6, 201 (2018)

43. Dutta, B., Guha, D.: Partitioned Bonferroni mean based on linguistic 2-tuple for dealing with multi-attribute group decision making. Appl. Soft Comput. 37, 166-179 (2015)

44. Liu, P.D., Chen, S.M., Wang, Y.M.: Multiattribute group decision making based on intuitionistic fuzzy partitioned Maclaurin symmetric mean operators. Inf. Sci. 512, 830-854 (2019)

45. Chen, S.M., Tan, J.M.: Handling multicriteria fuzzy decision making problems based on vague set-theory. Fuzzy Sets Syst. 67, 163-172 (1994)

46. Hong, D.H., Choi, C.H.: Multicriteria fuzzy decision-making problems based on vague set theory. Fuzzy Sets Syst. 114, 103$113(2000)$
47. Pecaric, J., Wen, J., Wang, W.L., Lu, T.: A generalization of Maclaurin's inequalities and its applications. Math. Inequal. Appl. 8, 583-598 (2005)

48. Wang, X.T., Triantaphyllou, E.: Ranking irregularities when evaluating alternatives by using some Electre methods. Omega 36, 45-63 (2008)

49. Chen, Z.S., Yang, L.L., Rodrguez, M.R., Xiong, S.H., Chin, K.S., Martnez, L.: Power-average-operator-based hybrid multiattribute online product recommendation model for consumer decisionmaking. Int. J. Intell. Syst. 36, 2572-2617 (2021)

50. Mahmood, T., Ullah, K., Khan, Q., Jan, N.: An approach toward decision-making and medical diagnosis problems using the concept of spherical fuzzy sets. Neural Comput. Appl. 31, 7041-7053 (2019)

51. Ashraf, S., Abdullah, S.: Spherical aggregation operators and their application in multiattribute group decision-making. Int. J. Intell. Syst. 34, 493-523 (2019)

52. Garg, H.: A new generalized Pythagorean fuzzy information aggregation using Einstein operations and its application to decision making. Int. J. Intell. Syst. 31, 886-920 (2016)

53. Ali, M.I.: Another view on q-rung orthopair fuzzy sets. Int. J. Intell. Syst. 33, 2139-2153 (2018)

54. Khan, M.J., Ali, M.I., Kumam, P.: A new ranking technique for q-rung orthopair fuzzy values. Int. J. Intell. Syst. 36, 558-592 (2021)

55. Garg, H., Ali, Z., Mahmood, T., Aljahdali, S.: Some similarity and distance measures between complex interval-valued q-rung orthopair fuzzy sets based on cosine function and their applications. Math. Probl. Eng. (2021). https://doi.org/10.1155/2021/ 5534915

56. Batool, B., Ahmad, M., Abdullah, S., Ashraf, S., Chinram, R.: Entropy based Pythagorean probabilistic hesitant fuzzy decision making technique and its application for fog-haze factor assessment problem. Entropy 22, 318 (2020)

Publisher's note Springer Nature remains neutral with regard to jurisdictional claims in published maps and institutional affiliations. 